\title{
Asymptotic Completeness and Multiparticle Structure in Field Theories
}

\author{
D. Iagolnitzer ${ }^{1}$ and J. Magnen ${ }^{2}$ \\ ${ }^{1}$ Service de Physique Théorique, CEN Saclay, F-91191 Gif-sur-Yvette Cedex, France \\ ${ }^{2}$ Centre de Physique Théorique, Ecole Polytechnique, F-91128 Palaiseau Cedex, France
}

\begin{abstract}
Previous proofs of asymptotic completeness and related results on scattering in field theories are restricted to $P(\varphi)_{2}$ models in the 2- and 3-particle regions. In this paper, new cluster expansions that are well adapted to more direct proofs and generalizations of these results are presented. In contrast to previous ones, they are designed to provide direct graphical definitions of general irreducible kernels satisfying structure equations recently proposed and shown to be closely linked with asymptotic completeness and with the multiparticle structure of Green functions and collision amplitudes in general energy regions. The method can be applied as previously to $P(\varphi)_{2}$ and can also be extended to theories involving renormalization which are controlled by phase-space analysis. It is here illustrated in detail for the Bethe-Salpeter kernel in $\varphi_{2}^{4}$, in which case a new proof of its 4-particle decay (which yields asymptotic completeness in the 2-particle region) is given.
\end{abstract}

\section{Introduction}

\subsection{Background and General Ideas}

Past results on asymptotic completeness and related results in constructive theory apply to weakly coupled, superrenormalizable $P(\varphi)_{2}$ models in the 2-particle [1, 2] and 3-particle [3] region. (For some previous preliminary results related to spectrum see also [0].) In [1 a] the Bethe-Salpeter kernel $G$ is defined through the B.S. equation

$$
F=G+F \circ G
$$

where $F$ is the connected, amputated 4-point function and $F \circ G$ is a Feynman-type convolution integral ${ }_{2}^{1}=\mathrm{B}=\mathrm{G}={ }_{4}^{3}$ with 2-point functions on internal lines. $G$ is then shown to satisfy in Euclidean space-time 3-particle decay, or in an even theory (e.g. $\left.\varphi_{2}^{4}\right)$ 4-particle decay of the form $e^{-m(1-\varepsilon) d\left(x_{1}, \ldots, x_{4}\right)}$ with:

$$
d\left(x_{1}, \ldots, x_{4}\right)=4\left|\frac{x_{1,0}+x_{2,0}}{2}-\frac{x_{3,0}+x_{4,0}}{2}\right|+\left|x_{1,0}-x_{2,0}\right|+\left|x_{3,0}-x_{4,0}\right|,
$$


where $m>0$ is the bare mass (close to the physical mass $m_{\mathrm{ph}}$ in the models considered when the coupling constant $\lambda \rightarrow 0)$ and $\varepsilon \rightarrow 0$ as $\lambda \rightarrow 0$. Equivalent analyticity (=2-particle irreducibility) of $G$ follows in momentum-space, via the Laplace transform theorem, below the 4-particle threshold $\left[s<\left(4 m_{\mathrm{ph}}\right)^{2}-\varepsilon^{\prime}\right.$ in the even case, $\varepsilon^{\prime} \rightarrow 0$ as $\lambda \rightarrow 0$, where $s$ is the center of mass squared energy in Minkowski space).

It is then shown for the same models in $[5,1 \mathrm{~b}]$ that this analyticity of $G$, together with a related property of the 2-point function (isolated pole at the physical mass), yields asymptotic completeness e.g. in the form of the relation

$$
F_{+}-F_{-}=F_{+} * F_{-}
$$

in the 2-particle region $\left(2 m_{\mathrm{ph}}\right)^{2}<s<\left(4 m_{\mathrm{ph}}\right)^{2}-\varepsilon^{\prime}, \varepsilon^{\prime} \rightarrow 0$ as $\lambda \rightarrow 0$, where $*$ denotes onmass shell convolution over two internal energy-momenta. The existence of an analytic continuation of $F$ in a two-sheeted domain around the 2-particle threshold (with possible poles) is also established. The absence of pole in the physical sheet, hence of 2-particle bound state, at $s<\left(2 m_{\mathrm{ph}}\right)^{2}$ is obtained for $\varphi_{2}^{4}$. One such bound state is present for other $P(\varphi)_{2}$ models such as $\varphi_{2}^{6}-\varphi_{2}^{4}$ near $s=\left(2 m_{\mathrm{ph}}\right)^{2}$ [4]. In this connection, we note that a quasi-equivalence has been as a matter of fact established in a more general axiomatic framework, between the 2particle irreducibility of $G$ (and the related property of the 2-point function) and the asymptotic completeness equation (3), first in the earlier work [5] and in a more complete way in [6]. It yields, in particular, a general derivation of (3) from the irreducibility of $G$, apart from possible poles of $F$. The latter will be excluded by a general argument (in the 2-particle region) in [7], where the analysis of bound states and resonances for $P(\varphi)_{2}$ models and its extension to the Gross-Neveu model will also be given by an adaptation of the method of $[5,6]$. (A different technical approach was used in $[1,2,4]$.)

Another method in which a somewhat different type of irreducible kernel and Bethe-Salpeter equation is introduced, has been proposed and applied in the 2particle region in [2b]. The results above have been, on the other hand, extended in [3b] [for even $P(\varphi)_{2}$ models and up to some technical limitations] to the 3-particle region, a case in which significant complications already occur, but which still involves important simplifications with respect to more general energy regions (see [8]). The derivation of irreducibility of relevant kernels is obtained there by the method of [1]. The subsequent derivation of asymptotic completeness properties is linked with the analysis of [9] (given in the same 3-particle region in an even theory) which extends the previous works $[5,6]$, and makes use of a new integral equation involving the six-point function and a 3-particle irreducible kernel. It has been believed for a long time that more general results should be obtained in a way similar to above. However, important difficulties have been encountered in this program. A (limited) class of irreducible kernels and integral equations has been introduced in [2a, 3a, 3c] (see also [9]). A more heuristic analysis has then been proposed in [8] where structure equations, involving more general irreducible kernels, that are generalizations, in each energy region, of the Neumann series of $F$ in terms of $G$, and from which suitable sets of integral equations might be ultimately derived, have been conjectured. Together with some mathematical conjectures, they have, moreover, been shown, at least in a formal sense (thus for 
theories without bound states), to yield asymptotic completeness relations in general energy regions, as also further results on the multiparticle analytic structure of Green functions and collision amplitudes.

In this work, the definition of irreducible kernels and the derivation of irreducibility are reconsidered. First, " $p^{\text {th }}$ order" cluster expansions that are simple generalizations of previous ones are introduced. (A similar expansion of order 3 is used in [10] for mass renormalization purposes.) They yield, with $p=4$, a corresponding explicit graphical definition of $G$ and a new, direct proof of its 4particle decay. By direct graphical inspection, $G$ is known, on the other hand, to satisfy the Bethe-Salpeter equation (1). The analysis is presented for simplicity for $\varphi_{2}^{4}$, but applies also to other $P(\varphi)_{2}$ models. The type of expansion of $G$ that is introduced has the advantage, in contrast to the method of [1], of giving a direct graphical interpretation of irreducibility very close to that of perturbation theory, and can be easily adapted for $p$ large enough to the definition (and proof of irreducibility properties) of general irreducible kernels satisfying the structure equations of [8]. In fact, this expansion exhibits $G$ as an infinite sum of terms associated with 2-particle (or 3-particle in the even case) irreducible graphs in space-time that join squares of a unit lattice containing $x_{1}, \ldots, x_{4}$ and possibly intermediate arbitrary squares. A propagator, that yields an exponential fall-off factor $e^{-(m-\varepsilon)|l|}$, is associated to each line $l$ of the graph. An exponential fall-off in $e^{-m\left(1-\varepsilon^{\prime}\right) l_{3}\left(x_{1}, \ldots, x_{4}\right)}$, where $\varepsilon^{\prime}>\varepsilon$ and $l_{3}\left(x_{1}, x_{2}, x_{3}, x_{4}\right)$ is the shortest length of all 3particle irreducible graphs joining $x_{1}, \ldots, x_{4}$ and possibly other points is then obtained. This fall-off, which generalizes the tree-graph decay introduced previously for connected functions in statistical mechanics in [11] and in constructive theory in [12], is stronger than the decay factor (2) (which is sufficient for present purposes). The factor $e^{-m\left(1-\varepsilon^{\prime}\right) l_{3}}$ is first extracted for all terms of the expansion of $G$. Remaining factors $e^{-m\left(\varepsilon^{\prime}-\varepsilon\right)|l|}$ for each line are then shown to ensure convergence at small values of the coupling constant $\lambda$ (first in a finite volume $\Lambda$ and then in the $A \rightarrow \infty$ limit). The convergence of present expansions of $F$ and $G$, in contrast to the divergence of the perturbative series, is due to the suitable use of a lattice. In the perturbative series, each square of the lattice contains an arbitrary number of vertices. Hence there is an arbitrary number of propagators between any pair of squares, whereas only a finite number $(\leqq p)$ of propagators are made explicit between squares in the cluster expansions, which are in fact "minimal" expansions with respect to the lattice.

In Part II of this work (to be published elsewhere) the method will be extended to theories involving renormalization, hence in which the usual B.S. equation (1) does not make sense (divergence of $F \circ G$ ), and more precisely to the massive GrossNeveu model (in dimension 2) whose existence has been recently established [10, 13]. The result then applies to a kernel $G_{M}$ satisfying a regularized, nonrenormalized, B.S. type equation:

$$
F=G_{M}+F{ }_{M} G_{M},
$$

where ${ }^{\circ}$ is defined as $\circ$, but (besides 2-point functions) well specified analytic cut-off factors with sufficient decrease at infinity in Euclidean directions are attached to each internal line in momentum-space. The 4-particle decay of $G_{M}$ and Eq. (4) are now obtained through phase-space expansions of $F$ and $G_{M}$ that 
extend those introduced previously. (A $4^{\text {th }}$-order cluster expansions is used in the slice of lowest momentum, with still $1^{\text {st }}$ order expansions in higher slices.) Kernels satisfying a regularized equation of type (4) are those most appropriate for the derivation of asymptotic completeness: in fact, the analysis of $[5,6]$ applies equally in this case, and was indeed made in terms of kernels $G_{\alpha}$ satisfying this type of equation. An alternative approach using Bether-Salpeter kernels $G_{\alpha}^{\prime}$ in a theory with cut-off (satisfying $F_{\alpha}=G_{\alpha}^{\prime}+F_{\alpha}{ }_{\alpha} G_{\alpha}^{\prime}$ ) would introduce problems in the limit when the cut-off is removed. The (non-simple) perturbative content of the kernels $G_{M}$ and their connection with the kernels $G_{\alpha}^{\prime}$ and with the "renormalized" irreducible kernel $G_{\text {ren }}$ (i.e. from the perturbative viewpoint the formal sum of renormalized 2-particle irreducible graphs) have been indicated in [14]. This will be made precise in the framework of constructive theory in [15], where renormalized B.S. equations of the type proposed in $[16]$ and in $[17,14]$ will also be established in that context. As explained in [15], renormalized kernels and B.S. equations might be used alternatively to obtain 2-particle asymptotic completeness for the G.N. model. However, this introduces unnecessary complications. The use of regularized kernels allows a more direct exploitation of the local analyticity (= irreducibility) properties in momentum space which are the important feature needed in the derivation of asymptotic completeness, whereas renormalized kernels and B.S. equations are also linked with asymptotic properties in momentum space (in Euclidean directions), i.e. with short distance behaviour in space-time (see [16, 17] and [15]).

The method is again well adapted to more general regions: it yields explicit definitions (and irreducibility properties) of general irreducible kernels satisfying again (by a graphical analysis analogous to that of [8]) regularized structure equations of the type conjectured there and shown as before to be closely linked with asymptotic completeness and with the multiparticle structure in general energy regions. More details in general energy regions will be given elsewhere both for $P(\varphi)_{2}$ and for the Gross-Neveu model. All results apply, as those of [1-3], for small couplings, although similar results can be expected in pure phases at large values of $\lambda$.

The paper will include a self-contained presentation of previous results, such as the existence of $\varphi_{2}^{4}$ and (in Part II) of the G.N. model. This presentation differs from the earlier works on some technical points and includes on these points some simplifications and improvements. Contents are described in Sect. 1.2. More specific introductory comments will be given in Sect. 2 .

\subsection{Description of Contents}

The model is defined in Sect. 2. Connected functions $H_{\Lambda}^{c}\left(z_{1}, \ldots, z_{l}\right)$ are first defined in Euclidean space-time in a finite box $\Lambda$. The non-connected function $H_{\Lambda}\left(z_{1}, \ldots, z_{l}\right)$, is equal to $Z_{\Lambda}{ }^{-1} I_{\Lambda}\left(z_{1}, \ldots, z_{l}\right)$, where $Z_{\Lambda}$ is a normalization factor. Cluster expansions of order $p$ of $I_{\Lambda}, p \geqq 1$, are introduced in Sect. 3 by a generalization of a cluster expansion due to $[18,19]$, which is more simple and convenient than previous ones [20]. They provide expansions of the form:

$$
I_{\Lambda}\left(z_{1}, \ldots, z_{l}\right)=\sum_{q \geqq 1} \sum_{X_{1}, \ldots, X_{q}} \prod_{i=1}^{q} I\left(X_{i} ;\left[z_{r}\right]_{i}\right)
$$


where the sum runs over all partitions of the set of unit squares of $\Lambda$ into subsets $X_{1}, \ldots, X_{q} . I\left(X_{i}\right)$, which depends on the set $\left[z_{r}\right]_{i}$ of variables $z_{r}$ in $X_{i}$, has the form:

$$
I(X)=\sum_{G} I(X, G),
$$

where the sum runs over a class of connected graphs $G$ linking the squares of $X$, which in the cluster expansion of order $p$ have at most $p$ lines between any pair of squares of $X$. Each line is associated with a propagator $C$. Bounds on $|I(X, G)|$, including fall-off factors $e^{-(m-\varepsilon) d(l)}$ for each line $l$ of $G$ are then exhibited. If $X$ is composed of only one square $\Delta$ containing no point $z_{r}, I(X)$ is equal to a constant $a \neq 0$ independent of $\Delta$ and (5) can thus be written in the form:

$$
a^{-|\Lambda|} I_{\Lambda}\left(z_{1}, \ldots, z_{l}\right)=\sum_{q \geqq 1} \sum_{X_{1}, \ldots, X_{q}}^{\prime} \prod_{i=1}^{q}\left(I\left(X_{i}\right) a^{-\left|X_{i}\right|}\right),
$$

where the sum runs now over $q \geqq 1$ (non-overlapping) subsets $X_{1}, \ldots, X_{q}, q \geqq 1$, of squares of $\Lambda$ such that $\left|X_{i}\right| \geqq 2$ or $\left|X_{i}\right|=1, z_{r} \in X_{i}$ for at least one $r$.

On the other hand, $I(X, G)$ and hence $I(X)$ satisfy, for $p>1$, factorization properties that will be useful in the study of irreducible kernels. In fact, if $G$ can be divided into two subgraphs $G^{\prime}, G^{\prime \prime}$ by cutting a set $S$ of $p^{\prime}<p$ lines, then $I(X, G)$ factorizes in a natural way as a convolution product, with propagators on each line of $S$, of functions associated with $X^{\prime}, G^{\prime}$ and $X^{\prime \prime}, G^{\prime \prime}$, respectively $\left(X^{\prime}\right.$, respectively $X^{\prime \prime}$, is the set of squares of $X$ linked by $G^{\prime}$, respectively $\left.G^{\prime \prime}\right)$. More generally, a similar factorization property follows if $G$ is decomposed into its " $(p-1)$ irreducible" components: by definition, a subgraph is $(p-1)$-irreducible (in all channels) if it cannot be divided into two parts by cutting less than $p$ lines, whereas each $(p-1)$-irreducible component can be separated successively from the rest of the graph by cutting less than $p$ lines. It yields the formula:

$$
\begin{aligned}
I\left(X ;\left[z_{r}\right]\right)= & \left.\sum_{\substack{\text { partitions of } X \\
\text { into subsets } X_{1}, \ldots, X_{q} \\
q \geqq 1}} \sum_{G}\left(n_{\alpha, \beta} !\right)^{-1}\right) \int \prod_{l \in G} d u_{l} d v_{l} C\left(u_{l}, v_{l}\right) \\
& \times \prod_{j=1}^{q} \hat{I}_{(\text {irr) }}\left(X_{j} ;\left[z_{r}\right]_{j},[u, v]_{j}\right),
\end{aligned}
$$

where the second sum runs over graphs $\tilde{G}$ whose "vertices" are the sets $X_{1}, \ldots, X_{q}$ and which contain no $(p-1)$-irreducible part, the points $u_{l}, v_{l}$ are integrated in the respective sets $X_{\alpha}, X_{\beta}$ joined by line $l, n_{\alpha, \beta}$ is the number of lines between $X_{\alpha}$ and $X_{\beta}$, and the function $\hat{I}_{\text {(irr) }}(X)$ is the sum of all terms $\hat{I}(X, G)$ corresponding to $(p-1)$ irreducible graphs. $[\hat{I}(X, G)$ is defined in a way analogous to $I(X, G)$, but the dependence on the variables $u, v$ in $X$ is somewhat different.]

More precisely, two different types of cluster expansions will be presented. They both provide Eqs. (5)-(8) with the same functions $I(X), \hat{I}_{\mathrm{irr}}(X)$, which are in fact defined by Eqs. (5), (8), but with a different class of graphs $G$ and different functions $I(X, G)$. The first one, whose description is the most simple, is described in Sect. 3.1. It can be used in a natural way in Part II for the Gross-Neveu model. On the other hand, as will be discussed, its use is possible but not satisfactory for bosonic models like $P(\varphi)_{2}$. We then present, in Sect. 3.2, alternative $p^{\text {th }}$ order expansions. For any $p \geqq 1$, they can be constructed in various ways depending 
on the choice of a first square and of some subsequent ones. Individual terms $I(X, G)$ depend on this choice. However, bounds used later do not depend on it: see Sect. 3.3.

In Sect. 4, an à la Mayer procedure analogous to that of [12] is used to derive corresponding $p^{\text {th }}$-order expansions of $H_{A}=Z_{\Lambda}^{-1} I_{\Lambda}$ and in turn of $H_{\Lambda}^{c}$. If $z_{1}, \ldots, z_{l}$ belong to different squares, the latter is of the form:

$$
H_{\Lambda}^{c}\left(z_{1}, \ldots, z_{l}\right)=\sum_{N} \frac{1}{N !} \sum_{\substack{\Delta_{1}, \ldots, \Delta_{N} \\ \Delta_{i} \subset \Lambda, i=1, \ldots, N}} \sum_{\mathbf{G}} \mathbf{I}(X, \mathbf{G}),
$$

where $X$ is the set of squares $\Delta_{1}, \ldots, \Delta_{N}$ and of the squares $\Delta_{0,1}, \ldots, \Delta_{0, l}$ containing $z_{1}, \ldots, z_{l}$, and the last sum runs over connected graphs $\mathbf{G}$ made up of subgraphs $G$ and of further Mayer lines joining pairs of squares of $X$ : a Mayer line indicates that the two squares must coincide and gives a factor -1 . (In the absence of a Mayer line, squares vary independently in $A$ and are allowed to coincide. The graphs $\mathbf{G}$ are connected when all lines, including Mayer lines, are taken into account.) In view of the previous bounds on $|I(X, G)|$, it is then shown in Sect. 4 (after a suitable regrouping of terms associated with graphs $\mathbf{G}$ with different sets of Mayer lines) that the expansion (9) is uniformly convergent at small enough values of $\lambda$. The convergence is uniform with respect to $\Lambda$ and $H_{\Lambda}^{c}$ is well defined in the $\Lambda \rightarrow \infty$ limit. The method also allows one to obtain (for $H_{A}^{c}$ and $H^{c}$ ) a decay factor $e^{-m(l-\varepsilon) l_{0}\left(z_{1}, \ldots, z_{l}\right)}$, where $l_{0}$ is the shortest length of connected graphs joining $z_{1}, \ldots, z_{l}$ and possibly other points (tree-decay). Although the proof requires only $p=1$ expansions, it will be explained on $p^{\text {th }}$-order ones in view of later extensions.

In Sect. 5, the kernel $G$ is simply defined as the partial sum, in the $4^{\text {th }}$-order expansion of the amputated, connected function $F\left(z_{1}, \ldots, z_{4}\right)$, corresponding to graphs $\mathbf{G}$ that are 3-particle irreducible in the $2 \rightarrow 2$ channel considered (and 2-particle irreducible in $1 \rightarrow 3$ channels; each Mayer line is to be considered as "infinitely irreducible"). This definition ensures, in view of the factorization properties of type (8), that $G$ does satisfy the Bethe-Salpeter equation (1). The convergence and decay properties of $G$ follow from an adaptation of the methods used in Sect. 4. Section 5 also contains the fully analogous analysis (needed in Sect. 6) of the 1-particle irreducible 2-point function $K$. The momentum-space analyticity properties of $G$ and of the (complete) 2-point function, needed in the derivation of 2-particle asymptotic completeness, are finally given in Sect. 6.

\section{The Model}

The non-connected 4-point Green-function $S_{\Lambda}\left(x_{1}, \ldots, x_{4}\right)$ of $\varphi_{2}^{4}$ can be written in a box $\Lambda$ as $Z_{\Lambda}^{-1} N_{\Lambda}\left(x_{1}, \ldots, x_{4}\right)$, where:

$$
\begin{gathered}
Z_{\Lambda}=\lim _{\varrho \rightarrow \infty} \int e^{-\lambda f: \varphi^{4}:_{\varrho}(x) d x} d \mu_{\varrho}(\varphi), \\
N_{\Lambda}\left(x_{1}, \ldots, x_{4}\right)=\lim _{\varrho \rightarrow \infty} \int \varphi\left(x_{1}\right) \ldots \varphi\left(x_{4}\right) e^{-\lambda \int: \varphi^{4}:_{\varrho}(x) d x} d \mu_{\varrho}(\varphi),
\end{gathered}
$$

where $: \varphi^{4}::_{\varrho}(x)=\varphi^{4}(x)-6 C_{\varrho}(0,0) \varphi^{2}(x)+3 C_{\varrho}(0,0)^{2}$ and $C_{\varrho}(x, y)$ is the Fourier transform of the momentum space regularized propagator 
$\left(p^{2}+m^{2}\right)^{-1} \exp \left[-M^{-2 \varrho}\left(p^{2}+m^{2}\right)\right] ; d \mu_{\varrho}$ is the measure of covariance $C_{\varrho}$. From now on, we leave implicit the dependence in $\varrho$. For the simple and well known treatment of the $\varrho \rightarrow \infty$ limit, see e.g. [21, 22]. For our purposes, it is convenient to "integrate by parts" the fields $\varphi(x)$ in (11), i.e. to replace each field $\varphi\left(x_{i}\right), i=1, \ldots, 4$, by $\int d z_{i} C\left(x_{i}-z_{i}\right) \frac{\delta}{\delta \varphi\left(z_{i}\right)}$. This gives:

$$
N_{\Lambda}\left(x_{1}, \ldots, x_{4}\right)=\int_{\substack{z_{i} \subset \Lambda \\ i=1, \ldots, 4}} d z_{1} \ldots d z_{4} \prod_{i=1}^{4} C\left(x_{i}-z_{i}\right) N_{\Lambda}^{\prime}\left(z_{1}, \ldots, z_{4}\right)+R_{\Lambda}\left(x_{1}, \ldots, x_{4}\right)
$$

where

$$
N_{\Lambda}^{\prime}\left(z_{1}, \ldots, z_{4}\right)=\int \prod_{i=1}^{4} \frac{\delta}{\delta \varphi\left(z_{i}\right)} e^{-\lambda \int_{\Lambda}: \varphi^{4}:(x) d x} d \mu(\varphi),
$$

and $R_{A}$ is a sum of terms of the form $C\left(x_{i}-x_{j}\right) C\left(x_{k}-x_{l}\right)$ and $C\left(x_{i}-x_{j}\right)$

$$
\int d z_{k} d z_{l} C\left(x_{k}-z_{k}\right) C\left(x_{l}-z_{l}\right) N_{\Lambda}^{\prime}\left(z_{k}, z_{l}\right), \quad[i, j, k, l]=[1,2,3,4],
$$

which will not contribute to the connected 4-point function. By explicit calculation, $N_{A}^{\prime}$ can, on the other hand, be written in the form:

$$
\begin{aligned}
N_{\Lambda}^{\prime}\left(z_{1}, \ldots, z_{4}\right)= & \delta\left(z_{1}-z_{2}\right) \delta\left(z_{1}-z_{3}\right) \delta\left(z_{1}-z_{4}\right) \lambda Z_{A} \\
& +\delta\left(z_{1}-z_{2}\right) \delta\left(z_{1}-z_{3}\right) I_{\Lambda}^{(1,3)}\left(z_{1}, z_{4}\right)+\ldots \\
& +\delta\left(z_{1}-z_{2}\right) \delta\left(z_{3}-z_{4}\right) I_{\Lambda}^{(2,2)}\left(z_{1}, z_{3}\right)+\ldots \\
& +\delta\left(z_{1}-z_{2}\right) I_{\Lambda}^{(2,3,3)}\left(z_{1}, z_{3}, z_{4}\right)+\ldots+I_{A}^{(3,3,3,3)}\left(z_{1}, z_{2}, z_{3}, z_{4}\right)
\end{aligned}
$$

where the dots refer to similar terms obtained by permutations of indices and:

We finally define:

$$
I_{A}^{\left(n_{1}, \ldots, n_{l}\right)}\left(z_{1}, \ldots, z_{l}\right)=\int_{\substack{z_{i} \in A \\ i=1, \ldots, l}} \prod_{i=1}^{l}\left[-\left(4 ! / n_{i} !\right) \lambda: \varphi^{n_{i}}:\left(z_{i}\right)\right] e^{-\lambda f: \varphi^{4}:(x) d x} d \mu(\varphi) .
$$

$$
\begin{gathered}
H_{\Lambda}\left(z_{1}, \ldots, z_{l}\right)=Z_{\Lambda}^{-1} I_{\Lambda}\left(z_{1}, \ldots, z_{l}\right), \\
H_{\Lambda}^{c}\left(z_{1}, \ldots, z_{l}\right)=\text { connected part of } H_{\Lambda}\left(z_{1}, \ldots, z_{l}\right),
\end{gathered}
$$

where the indices $n_{i}$ have been left implicit, and state the following result [12] (previously proposed with a weaker type of fall-off in [20]), which will be reobtained in Sect. 3.

Proposition 1. $H_{\Lambda}^{c}$ has a well defined limit $H^{c}$ when $\Lambda \rightarrow \infty$. Moreover, $\forall \varepsilon>0, \exists \lambda_{\varepsilon}>0$ and $C_{\varepsilon}$ (independent of $\Lambda$ ) such that for any ( finite or infinite) $\Lambda$ and any $\lambda,|\lambda| \leqq \lambda_{\varepsilon}$ :

$$
\left.\left|H_{\Lambda}^{c}\left(z_{1}, \ldots, z_{l}\right)\right|<|\lambda|^{l} C_{\varepsilon} e^{-(m-\varepsilon) l_{0}\left(z_{1}, \ldots, z_{l}\right)}\left(1+\left|\ln \operatorname{Inf}_{i \neq j}\right| z_{i}-z_{j} \mid\right) \mid\right)^{6} .
$$

The length $l_{0}\left(z_{1}, \ldots, z_{l}\right)$ is, as already mentioned, the minimal length of all connected trees joining $z_{1}, \ldots, z_{l}$ and possibly intermediate points. The last factor $\left(1+\left|\left(\ln \operatorname{Inf}\left|z_{1}-z_{j}\right|\right)\right|\right)^{6}$ is included to take into account the short-distance behaviour of $H_{A}^{c}$, but it can be removed if one considers, e.g. the region $\left|z_{i}-z_{j}\right|>1, \forall(i, j), i \neq j$.

Proposition 1 yields corresponding results on the connected 4-point function $S_{\Lambda}^{c}$. 


\section{3. $p^{\text {th }}$-Order Cluster Expansions}

\subsection{Pairwise Cluster Expansions}

The most simple type of $p^{\text {th }}$-order cluster expansion that might be thought of is as follows. Auxiliary variables $s_{\Delta, \Delta^{\prime}}$ are attached to each pair of squares of $\Lambda$ and $C(x, y, s), s=\left\{s_{\Delta, \Delta^{\prime}}\right\}$, is defined as $s_{\Delta, \Delta^{\prime}} C(x, y)$ if $x \in \Delta, y \in \Delta^{\prime}, \Delta \neq \Delta^{\prime}, C(x, y, s)=C(x, y)$ if $x, y$ belong to a common square:

$$
C(x, y ; s)=C(x, y)\left[\sum_{\substack{\left(\Delta, \Delta^{\prime}\right) \\ \Delta \neq \Delta^{\prime}}} s_{\Delta, \Delta^{\prime}}\left(\Delta(x) \Delta^{\prime}(y)+\Delta^{\prime}(x) \Delta(y)\right)+\sum_{\Delta} \Delta(x) \Delta(y)\right],
$$

where $\Delta(x)=1$ if $x \in \Delta, \Delta(x)=0$ if $x \notin \Delta$. We note, in particular, that $C(x, y ; 1)$ $\equiv C(x, y), C(x, y ; s)=0$ if $x$ and $y$ belong to squares $\Delta, \Delta^{\prime}$ such that $s_{\Delta, \Delta^{\prime}}=0$, and $\frac{d}{d s_{\Delta, \Delta^{\prime}}} C(x, y ; s)=0$ unless $x \in \Delta, y \in \Delta^{\prime}$ or vice versa, in which case it is equal to $C(x, y)$. Let $I_{A}(s)$ be defined, formally, by replacing $C$ by $C(s)$ is the measure $d \mu$. Being given a partition of $\Lambda$ into subsets $X_{1}, \ldots, X_{q}$, if all the variables $s_{\Delta, \Delta^{\prime}}$ associated to squares $\Delta, \Delta^{\prime}$ that belong to different sets are fixed at zero, the measure $d \mu$ correspondingly factorizes. Then, by Taylor expansion of order $p$ of $I_{A}(s)$ :

$$
\begin{aligned}
I_{\Lambda} \equiv & I_{\Lambda}(1)=\left\{\prod _ { ( \Delta , \Delta ^ { \prime } ) } \left[\left|s_{\Delta, \Delta^{\prime}=0}+\frac{d}{d s_{\Delta, \Delta^{\prime}}}\right| s_{\Delta, \Delta^{\prime}=0}+\ldots+\frac{1}{(p-1) !} \frac{d}{d s^{p-1}} \mid s_{\Delta, \Delta^{\prime}=0}\right.\right. \\
& \left.\left.+\frac{1}{(p-1) !} \int_{0}^{1}\left(1-s_{\Delta, \Delta^{\prime}}\right)^{p-1} \frac{d^{p}}{d s_{\Delta, \Delta^{\prime}}^{p}}\right]\right\} I_{\Lambda}(s) .
\end{aligned}
$$

Expansions of $I_{A}$ of the form (5) to (7) are obtained with a sum in (6) over all connected graphs $G$ including $n_{\Delta, \Delta^{\prime}} \leqq p$ lines between any pair $\left(\Delta, \Delta^{\prime}\right)$ of squares of $X$ and:

$$
\begin{aligned}
& I(X, G)=\prod_{\substack{\left(\Delta, \Delta^{\prime}\right) \in X \\
n_{\Delta, \Delta^{\prime}} \leqq p-1}} \frac{1}{n_{\Delta, \Delta^{\prime}} !} \prod_{\substack{\Delta, \Delta^{\prime} \\
n_{\Delta, \Delta^{\prime}}=p}} \frac{1}{(p-1) !} \int_{0}^{1}\left(1-s_{\Delta, \Delta^{\prime}}\right)^{p-1} d s_{\Delta, \Delta^{\prime}} \\
& \times \int \prod_{l \in G} d u_{l} d v_{l}\left[\Delta_{l}\left(u_{l}\right) \Delta_{l}^{\prime}\left(v_{l}\right)+\Delta_{l}\left(v_{l}\right) \Delta_{l}^{\prime}\left(u_{l}\right)\right] \\
& \times \frac{\delta}{\delta \varphi\left(u_{l}\right)} \frac{\delta}{\delta \varphi\left(v_{l}\right)} \prod_{\substack{r=1, \ldots ., 1 \\
\text { such that } \\
z_{r} \in X}}\left(\lambda: \varphi^{n_{r}}:\left(z_{r}\right)\right) \\
& \times\left. e^{-\lambda \int: \varphi^{4}:(x) d x} d \mu(\varphi ; s)\right|_{s_{\Delta, \Delta^{\prime}}=0 \text { if } n_{\Delta, \Delta^{\prime}}<p} .
\end{aligned}
$$

The results above are only formal, as was already known at $p=1$ : the propagator $C(x, y ; s)$ is not in general of a positive type and the measure $d \mu(s)$ is thus not well defined in general. Various possibilities have then been considered at $p=1$. In a first one [23] a formula of type (18) is applied to $C^{1 / 2}$ (the square-root of $C$ in the operator sense) rather than to $C$. This allows one to avoid the previous difficulty. It introduces, however, a number of unpleasant features and, in particular, would not allow a direct analysis of the structure of the 2-point function (isolated pole at the 
physical mass), as that given in Sects. 5, 6. On the other hand, an alternative expansion, in which variables $s_{\Delta, \Delta^{\prime}}$ between squares are replaced by similar variables, first between a given square and its complement in $\Lambda$ and so forth, has been introduced in $[18,19]$ : this is the type of expansion that is generalized in Sect. 3. Although it introduces some arbitrariness it is a natural and simple way of solving the positivity problem: all $C(x, y ; s)$ either in $[18,19]$ or in Sect. 3 are by construction of a positive type, as linear combinations, with $\geqq 0$ coefficients, of positive-type terms.

We finally mention another way of solving the positivity problem, proposed in [24]: it is shown there that $C(x, y ; s)$ as defined in (18) can be of a positive-type if the side of the squares of the lattice is taken sufficiently large (and not of order 1 or $m^{-1}$ ) and if characteristic functions of these squares are replaced by smooth enough functions. This method introduces, however, at least from a conceptual viewpoint, an important loss of information.

\subsection{Inductive Cluster Expansions}

The cluster expansions of $I_{\Lambda}$ and $Z_{\Lambda}$ are obtained by introducing a lattice with squares of e.g. unit side in (2-dimensional) space-time (best results are obtained in principle when the side is of the order of $m^{-1}$ ) and auxiliary real variables as follows. First, a given square $\Delta_{1}$ is considered and $C\left(x, y ; s_{1}\right)$ is defined, for $0 \leqq s_{1} \leqq 1$, as

$$
C\left(x, y ; s_{1}\right)=C(x, y)\left[s_{1}+\left(1-s_{1}\right)\left\{\Delta_{1}(x) \Delta_{1}(y)+\left(1-\Delta_{1}\right)(x)\left(1-\Delta_{1}\right)(y)\right)\right],
$$

where $\Delta_{1}(x)$ is the characteristic function of $\Delta_{1}\left[\Delta_{1}(x)=1\right.$ if $x \in \Delta_{1}, \Delta_{1}(x)=0$ if $x \notin \Delta_{1}$ ]. I.e., $C\left(x, y ; s_{1}\right)=C(x, y)$ if $x$ and $y$ both belong to $\Delta_{1}$ or both belong to $\Lambda / \Delta_{1}$, and $C\left(x, y ; s_{1}\right)=s_{1} C(x, y)$ otherwise. In particular, $C(x, y ; 0)=0$ if $x \in \Delta_{1}, y \notin \Delta_{1}$ or vice versa. We also note that $\frac{d C}{d s_{1}}$ is equal to zero or to $C(x, y)$ in the first and second case, respectively. Functions $Z_{\Lambda}\left(s_{1}\right)$ and $I_{\Lambda}\left(z_{1}, \ldots, z_{l} ; s_{1}\right)$ are defined by formulae (10), (15) with $C$ replaced by $C\left(s_{1}\right)$ in the measure $d \mu$. We treat below $I_{\Lambda}, Z_{\Lambda}$ being treated similarly. A $1^{\text {st }}$ order Taylor formula gives (leaving $\Lambda$ implicit):

$$
I\left(z_{1}, \ldots, z_{l}\right) \equiv I\left(z_{1}, \ldots, z_{l} ; 1\right)=I\left(z_{1}, \ldots, z_{l} ; 0\right)+\int_{0}^{1} d s_{1} \frac{d}{d s_{1}} I\left(s_{1}\right) .
$$

In view of the previous property of $C(x, y ; 0)$, the measure $d \mu$ at $s_{1}=0$ factorizes, $\Delta_{1}$ and $\Lambda \backslash \Delta_{1}$ are decoupled and $I(0)$ reduces to the product $I_{\Delta_{1}} I_{\Lambda \backslash \Delta_{1}}$ (each factor depending on the variables $z$ in $\Delta_{1}$, respectively in $\left.\Lambda \backslash \Delta_{1}\right)$. On the other hand, $\frac{d I}{d s_{1}}$ can be written (in view of the property of $\left.\frac{d C}{d s_{1}}\right)$ as:

$$
\begin{gathered}
\frac{d I}{d s_{1}}\left(z_{1}, \ldots, z_{l} ; s_{1}\right)=\sum_{\Delta_{1}, 1 \neq \Delta_{1}} I_{\Delta_{1}, \Delta_{1}, 1}\left(z_{1}, \ldots, z_{l} ; s_{1}\right), \\
I_{\Delta_{1}, \Delta_{1,1}}\left(s_{1}\right)=\int d u d v\left[\Delta_{1}(u) \Delta_{1,1}(v)+\Delta_{1}(v) \Delta_{1,1}(u)\right] C(u, v) \frac{\delta}{\delta \varphi(u)} \frac{\delta}{\delta \varphi(v)} \\
\times \prod_{i=1}^{l}\left(\lambda: \varphi^{n_{i}}:\left(z_{i}\right)\right) e^{-\lambda \int: \varphi^{4}(x): d x} d \mu\left(\varphi ; s_{1}\right),
\end{gathered}
$$


where the integration in $\left(23^{\prime}\right)$ is restricted to $u$ in $\Delta_{1}, v$ in $\Delta_{1,1}$, or vice versa in view of $\Delta$-functions.

The term $I_{\Delta_{1}, \Delta_{1}, 1}\left(s_{1}\right)$ is represented schematically in Fig. 1, where the line between $\Delta_{1}$ and $\Delta_{1,1}$ represents the propagator $C(u, v)$.

Fig. 1

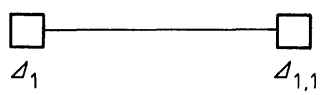

The term $I(0)$ is treated by applying to $I_{\Lambda \backslash \Delta_{1}}$ the same procedure as that presently described. For each value of $\Delta_{1,1}, I_{\Delta_{1}, \Delta_{1,1}}\left(s_{1}\right)$ is treated again by a $1^{\text {st }}$ order Taylor expansion:

$$
\begin{gathered}
I_{\Delta_{1}, \Delta_{1}, 1}\left(s_{1}\right)=I_{\Delta_{1}, \Delta_{1}, 1}(0)+\int_{0}^{s_{1}} \frac{d}{d s_{1}^{\prime}} I_{\Delta_{1}, \Delta_{1}, 1}\left(s_{1}^{\prime}\right) d s_{1}^{\prime}, \\
\frac{d}{d s_{1}^{\prime}} I_{\Delta_{1}, \Delta_{1,1}}\left(s_{1}^{\prime}\right)=\sum_{\Delta_{1,2} \neq \Delta_{1}} I_{\Delta_{1}, \Delta_{1,1}, \Delta_{1,2}}\left(s_{1}^{\prime}\right) .
\end{gathered}
$$

The measure $d \mu(\varphi ; 0)$ in $I_{\Delta_{1}, \Delta_{1}, 1}(0)$ is decoupled relatively to $\Delta_{1}$. Concerning the second term of (24), Taylor expansion is now applied to $I_{\Delta_{1}, \Delta_{1,1}, \Delta_{1,2}}\left(s_{1}^{\prime}\right)$. The procedure is pursued, until either one variable $s_{1}^{(\cdot)}$ is fixed at zero or $p$ squares among $\Delta_{1,1} \ldots \Delta_{1, r}$ coincide. We thus get at this stage either terms of the form $I_{\Delta_{1}, \Delta_{1}, 1, \ldots, \Delta_{1, r}}(0)$, in which case at most $p-1$ squares among $\Delta_{1,1} \ldots \Delta_{1, r}$ coincide and $\Delta_{1}$ is decoupled in the measure $d \mu$ from $\Lambda \backslash \Delta_{1}$ (but is linked by propagators to the squares $\Delta_{1,1} \ldots \Delta_{1, r}$ of $\left.\Lambda \backslash \Delta_{1}\right)$ or terms $I_{\Delta_{1}, \Delta_{1}, 1, \ldots, \Delta_{1, r}}\left(s_{1}^{(r-1)}\right)$, where $\Delta_{1, r}$ coincides with $p-1$ previous squares: see Fig. 2.

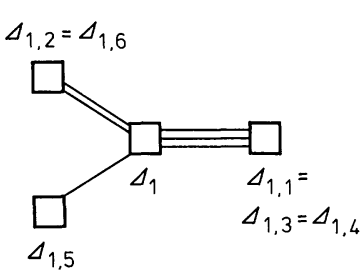

a) A term $I_{\Delta_{1}, \ldots, \Delta_{1, r}}(0)$

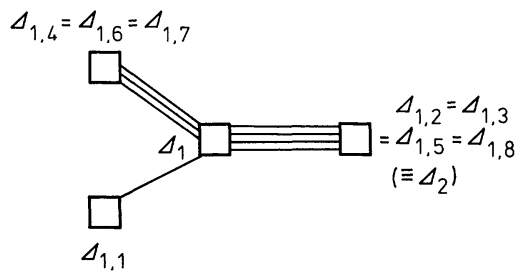

b) A term $I_{\left(p=4 \text { coincident squares } \Delta_{1, \imath}\right)}\left(s_{1}^{(r-1)}\right)$

Fig. 2. $(p=4)$

In the first case, the procedure to be applied is described later. In the second case, denoting below $\Delta_{2}$ the square $\Delta_{1, r}$, we now introduce a new variable $s_{2}$ relative to the set $\Delta_{1} \cup \Delta_{2}$ and the propagator:

$$
\begin{aligned}
& C\left(x, y ; s_{1}^{(r-1)}, s_{2}\right)=C\left(x, y ; s_{1}^{(r-1)}\right) \\
& \quad \times\left[s_{2}+\left(1-s_{2}\right)\left\{\left(\Delta_{1} \cup \Delta_{2}\right)(x)\left(\Delta_{1} \cup \Delta_{2}\right)(y)+\left(1-\left(\Delta_{1} \cup \Delta_{2}\right)(x)\right)\left(1-\Delta_{1} \cup \Delta_{2}\right)(y)\right\}\right],
\end{aligned}
$$

where $\Delta_{1} \cup \Delta_{2}(x)=\Delta_{1}(x)+\Delta_{2}(x)$, which yields a corresponding function $I_{\Delta_{1}, \Delta_{1}, 1, \ldots, \Delta_{1, r}}\left(s_{1}^{(r-1)}, s_{2}\right)$. This propagator satisfies properties similar to above, with $\Delta_{1}$ replaced by $\Delta_{1} \cup \Delta_{2}, \Lambda \backslash \Delta_{1}$ by $\Lambda \backslash\left(\Delta_{1} \cup \Delta_{2}\right), C(x, y)$ by $C\left(x, y ; s_{1}\right)$. We now consider $\left(1^{\text {st }}\right.$ order $)$ Taylor expansions with respect to $s_{2}, s_{2}^{\prime}, \ldots$ until either $s_{2}^{(q)}$ is 
fixed at zero for some $q\left(\Delta_{1} \cup \Delta_{2}\right)$ is then decoupled in the measure $d \mu$ from $\Lambda \backslash\left(\Delta_{1} \cup \Delta_{2}\right)$ or the square $\Delta_{2, r}$ coincides with $p-1$ previous squares among the squares $\Delta_{1, i} \neq \Delta_{2}$ and $\Delta_{2, j}, j<r$, i.e. this square, denoted $\Delta_{3}$ can be separated from $\Delta_{1} \cup \Delta_{2}$ only by cutting $p$ lines. (The dependence on $s_{2}^{(r-1)}$ is then kept.) We note that each square $\Delta_{2, j}$ is (in either case) linked by a propagator to $\Delta_{1} \cup \Delta_{2}$. The terms $I_{\Delta_{1}, \Delta_{1}, 1}, \ldots$ can be decomposed correspondingly into sums of integrals in which each $\Delta_{2, j}$ is linked by a propagator either to $\Delta_{1}$ or to $\Delta_{2}$. An example of a corresponding term (in the second case above, for $p=4$ ) is shown in Fig. 3.

Fig. 3

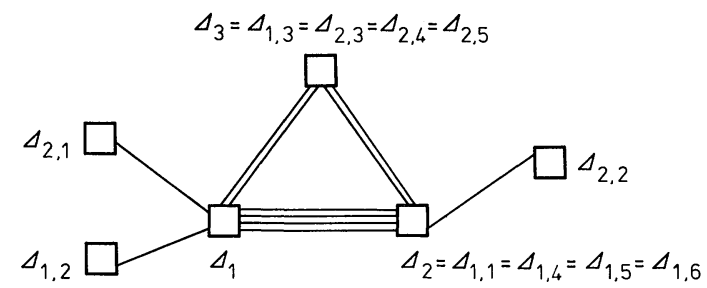

In the second case, a variable $s_{3}$ is then associated to the set $\left(\Delta_{1} \cup \Delta_{2}\right) \cup \Delta_{3}$ and so forth. The procedure is pursued until one variable $s_{k}^{(\cdot)}$ is fixed at zero. In this case, the measure $d \mu$ is decoupled relatively to $\Delta_{1} \cup \Delta_{2} \cup \ldots \cup \Delta_{k}$ and its complement in $\Lambda$. A square $\Delta_{k+1}$ is then chosen in $A \backslash\left(\Delta_{1} \cup \ldots \cup \Delta_{k}\right)$ and the same procedure as above is applied in $\Lambda \backslash\left(\Delta_{1} \cup \ldots \cup \Delta_{k}\right)$, with $\Delta_{1}, s_{1}$ replaced by $\Delta_{k+1}, s_{k+1}$, until either one variable $s_{k+1}^{(\cdot)}$ is fixed at zero or $\Delta_{k+1}$ is linked in a $(p-1)$-irreducible way to one or more squares of $\Lambda$ : i.e., the set formed by $\Delta_{k+1}$ and these squares, together with the lines that join them (associated with the various propagators already produced) can be separated into two parts only by cutting $p$ lines or more, but not less than $p$ lines. In e.g. the example of Fig. $2 \mathrm{a}(k=1)$, if $\Delta_{2}$ is chosen to coincide with $\Delta_{1,2}=\Delta_{1,6}$, a $(p-1)$-irreducible set (with $p=4$ ) is obtained if $\Delta_{2,1}$ and $\Delta_{2,2}$ coincide with $\Delta_{1,1}=\Delta_{1,3}=\Delta_{1,4}$. A new variable $s_{k+2}$ is then associated to the set $L$ and the procedure is pursued (with no line issued at this stage from the possible squares of $\Delta_{1} \cup \ldots \cup \Delta_{k}$ in $L$ ) until one $s_{k+2}^{(\cdot)}$ is fixed at zero or $L$ is contained in a larger irreducible set $L^{\prime}$, and so forth.

The final result is an expansion of $I$ of the form (5), (6), with:

$$
I(X, G)=\sum_{P} I(X, G, P),
$$

where the sum runs over a set of different procedures that give rise to the same graph $G$. Each term $I(X, G, P)$ is of the form:

where

$$
\begin{aligned}
I(X, G, P)= & \int_{0}^{1} d s_{1} \int_{0}^{s_{1}} d s_{1}^{\prime} \ldots \int_{0}^{s_{1}^{\left(r_{1}-2\right)}} d s_{1}^{\left(r_{1}-1\right)} \int_{0}^{1} d s_{2} \ldots \int_{0}^{s_{2}^{\left(r_{2}-2\right)}} d s_{2}^{\left(r_{2}-1\right)} \ldots \\
& \ldots M\left(s_{1}^{\left(r_{1}-1\right)}, s_{2}^{\left(r_{2}-1\right)}, \ldots\right) J\left(X, G, P ; s_{1}^{\left(r_{1}-1\right)}, s_{2}^{\left(r_{2}-1\right)}, \ldots\right),
\end{aligned}
$$

$$
\begin{aligned}
& J(X, G, P ; s)=\int d \mu(\varphi ; s) \\
& \quad \times \prod_{l \in G}\left\{\int d u_{l} d v_{l}\left[\Delta^{(l)}\left(u_{l}\right) \Delta^{\prime(l)}\left(v_{l}\right)+\Delta^{(l)}\left(v_{l}\right) \Delta^{(l)}\left(u_{l}\right)\right] C\left(u_{l}, v_{l}\right) \frac{\delta}{\delta \varphi\left(u_{l}\right)} \frac{\delta}{\delta \varphi\left(v_{l}\right)}\right\} \\
& \quad \times \prod_{r ; z_{r} \in X}\left(\lambda: \varphi^{\left.n_{r}:\left(z_{r}\right)\right) e^{-\lambda f: \varphi^{4}:(x) d x} .}\right.
\end{aligned}
$$


In (27), (28), $\Delta^{(l)}$ and $\Delta^{\prime(l)}$ are the squares linked by line $l$, and $M(s)$ is a product of variables $s_{i}$ at given powers coming from the dependence of some propagators on these variables [e.g. in the case of Fig. 3 , the propagator between $\Delta_{1}$ or $\Delta_{2}$ and $\Delta_{2, i}$, $i=1, \ldots, 5$ is $\left.C\left(u, v ; s_{1}^{(\cdot)}\right)=s_{1}^{(\cdot)} C(u, v)\right]$. We note that $d \mu(\varphi ; s)$ and $M(s)$ depend on $X, G, P$, although this dependence has been left implicit in (27), (28). In particular, some of the variables $s_{k}^{(\cdot)}$ may be fixed at zero in $d \mu(\varphi ; s)$, in which case $M$ and $d \mu$ factorize. This factorization occurs, in particular, in all cases when $G$ can be divided into two subgraphs $G^{\prime}, G^{\prime \prime}$ by cutting a set $S$ of $p^{\prime}<p$ lines, and then yields a corresponding expression of $J(X, G, P, s)$ as a convolution product, with propagators on each line of $S$, of functions $\hat{J}\left(X^{\prime}, G^{\prime}, P^{\prime} ;\left\{z_{r}\right\}^{\prime},\left\{u_{i}^{\prime}\right\}, s^{\prime}\right)$, $\widehat{J}\left(X^{\prime \prime}, G^{\prime \prime}, P^{\prime \prime} ;\left\{z_{r}\right\}^{\prime \prime},\left\{u_{i}^{\prime \prime}\right\}, s^{\prime \prime}\right)$. (Functions $\hat{I}, \widehat{J}$ are defined in a way analogous to $I, J$ with operators $\frac{\delta}{\delta \varphi\left(u_{l}\right)}$ attached to each variable $u_{l}$. The variables $u_{i}^{\prime}$ and $u_{i}^{\prime \prime}$ are integrated in $X^{\prime}$ and $X^{\prime \prime}$, respectively, and a factor $\left(p^{\prime} !\right)^{-1}$ is included.) $I(X, G, P)$ satisfies a similar property, but with factorized functions that still depend on the overall procedure $P$, as appears on the following example.

Example. Let $p=2,(X, G)=\stackrel{\Delta^{\prime \prime}}{\square} \stackrel{\Delta}{\square} \stackrel{\Delta^{\prime}}{\square}$ and $\Delta_{1}=\Delta$. Then 3 procedures $P_{1}, P_{2}, P_{3}$ are encountered:

$$
\begin{array}{ll}
P_{1}: & \Delta_{1,1}=\Delta^{\prime \prime}, \quad \Delta_{1,2}=\Delta_{1,3}=\Delta^{\prime}\left(=\Delta_{2}\right), \quad \Delta^{\prime \prime}=\Delta_{3}, \\
P_{2}: & \Delta_{1,1}=\Delta^{\prime}, \quad \Delta_{1,2}=\Delta^{\prime \prime}, \quad \Delta_{1,3}=\Delta^{\prime}\left(=\Delta_{2}\right), \quad \Delta^{\prime \prime}=\Delta_{3}, \\
P_{3}: & \Delta_{1,1}=\Delta_{1,2}=\Delta^{\prime}\left(=\Delta_{2}\right),
\end{array}
$$

with

$$
\begin{aligned}
I\left(X, G, P_{1}\right)= & I\left(X, G, P_{2}\right)=\int_{\substack{u \in \Delta \\
v \in \Delta^{\prime \prime}}} d u d v C(u, v) \\
& \times\left[\int_{0}^{1} d s_{1} \int_{0}^{s_{1}} d s_{1}^{\prime} \int_{0}^{s_{1}^{\prime}} d s_{1}^{\prime \prime} \hat{J}\left(\stackrel{\Delta}{\square} \stackrel{\Delta}{\square}^{\prime} ; u, s_{1}^{\prime \prime}\right)\right] \widehat{J}\left(\Delta^{\Delta^{\prime \prime}} ; v\right), \\
I\left(X, G, P_{3}\right)= & \int_{\substack{u \in \Delta \\
v \in \Delta^{\prime \prime}}} d u d v C(u, v) \\
& \times\left[\int_{0}^{1} d s_{1} \int_{0}^{s_{1}} d s_{1}^{\prime} s_{1}^{\prime} \hat{J}\left(\stackrel{\Delta}{\square} \square^{\Delta^{\prime}} ; u, s_{1}^{\prime}\right)\right]_{0}^{1} d s_{2} \hat{J}\left(\Delta^{\prime \prime} ; v\right),
\end{aligned}
$$

where the integral $\int_{0}^{1} d s_{2}$ in (30) can be removed ( $s_{2}$ fixed at zero in the last factor $\widehat{J}$ ).

On the other hand, the sum over procedures $P$ that give rise to the same set $\left(P^{\prime}, P^{\prime \prime}\right)$ does factorize in a natural way in terms of functions $\hat{I}\left(X^{\prime}, G^{\prime}, P^{\prime} ;\left\{z_{r}\right\}^{\prime},\left\{u_{i}^{\prime}\right\}\right)$, $\hat{I}\left(X^{\prime \prime}, G^{\prime \prime}, P^{\prime \prime} ;\left\{z_{r}\right\}^{\prime \prime},\left\{u_{i}^{\prime \prime}\right\}\right.$ ) (with as before propagators on each line of $S$ ). In the example above, there is only one pair $\left(P^{\prime}, P^{\prime \prime}\right)$ associated with $\Delta \cup \Delta^{\prime}$ and $\Delta^{\prime \prime}$, respectively $\left(P^{\prime}: \Delta_{1,1}=\Delta_{1,2}=\Delta^{\prime}=\Delta_{2}, P^{\prime \prime}\right.$ is trivial $)$. The result then comes from the identity (valid for any function $f$ ):

$$
2 \int_{0}^{1} d s_{1} \int_{0}^{s_{1}} d s_{1} \int_{0}^{s_{1}^{\prime}} d s_{1}^{\prime \prime} f\left(s_{1}^{\prime \prime}\right)+\int_{0}^{1} d s_{1} \int_{0}^{s_{1}} d s_{1}^{\prime} s_{1}^{\prime} f\left(s_{1}^{\prime}\right) \equiv \int_{0}^{1} d s_{1} \int_{0}^{s_{1}} d s_{1}^{\prime} f\left(s_{1}^{\prime}\right) .
$$


It follows more generally from similar identities (involving various sets of variables) proved directly by induction or checked at each order if $f(s)$ is replaced formally by $\sum_{n} f_{n} \prod_{k} s_{k}^{n_{k}}\left(n=\left\{n_{k}\right\}\right)$. The factorization of $I(X, G)$ mentioned in Sect. 1, and from there Eq. (8) follows.

Remarks. 1) As mentioned in Sect. $1, I(X, G)$ depends in general the choice of $\Delta_{1}$ and subsequent squares. An example at $p=2$ is

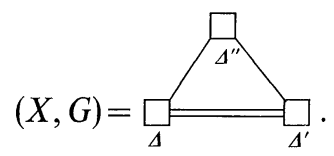

If $\Delta^{\prime \prime}=\Delta_{1}$, then either $\Delta=\Delta_{1,1}, \Delta^{\prime}=\Delta_{1,2}$ or vice versa. In either case, $s_{1}=0$ and $\Delta^{\prime \prime}$ is decoupled from $\Delta \cup \Delta^{\prime}$. If $\Delta=\Delta_{1}$, then $\Delta^{\prime}=\Delta_{2}, \Delta^{\prime \prime}=\Delta_{3}$ and $\Delta, \Delta^{\prime}, \Delta^{\prime \prime}$ remain coupled in the measure in all procedures. The corresponding terms $I(X, G, P)$ are different.

2) Some graphs $G$ cannot be obtained by any procedure $P$, in which case

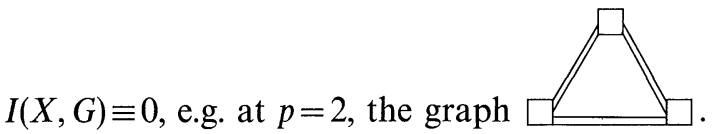

\subsection{Bounds on $I(X, G)$}

In view of the following lemma, which is an extension of a previous result at $p=1$ [19], the following bound holds on the terms $I(X, G)$ of Sect. 3.2:

$$
|I(X, G)|<\prod_{\left(v, v^{\prime}\right) \in G} \frac{1}{n_{v, v^{\prime}} !} \operatorname{Sup}_{P, s}|J(X, G, P, s)|,
$$

where $n_{v, v^{\prime}}$ is the number of lines joining the vertices $v, v^{\prime}$ of $G$, and bounds on $J$ independent of $P$ and $s$ will be obtained in view of the inequality $(0 \leqq)$ $C(x, y ; s) \leqq C(x, y)$.

\section{Lemma 1.}

$$
\begin{aligned}
\int d s M(s, G, P) & \equiv \sum_{P} \int_{0}^{1} d s_{1} \ldots \int_{0}^{s_{1}^{\left(r_{1}-2\right)}} d s_{1}^{\left(r_{1}-1\right)} \int_{0}^{1} d s_{2} \ldots \int d s_{2}^{\left(r_{2}-1\right)} \ldots M(s, G, P) \\
& =\prod_{\left(v, v^{\prime}\right) \in G}\left(n_{v, v^{\prime}} !\right)^{-1} .
\end{aligned}
$$

Proof. Let $F$ be the function of $\varepsilon=\left\{\varepsilon_{i, j}\right\}$, with one variable $\varepsilon_{i, j}$ for each pair $(i, j)$ of indices, $i=1, \ldots, n, j=1, \ldots, n, i \leqq j$, defined by:

$$
F(\varepsilon)=\prod_{(i, j)}\left(1+\varepsilon_{i, j}+\ldots+\varepsilon_{i, j}^{p}\right)=\sum_{\substack{\left\{n_{i j}\right\} \\ 0 \leqq n_{\imath}, j \leqq p}} \prod_{(i, j)}\left(\varepsilon_{i, j}\right)^{n_{\imath, j}} .
$$

The first expression of $F$ can be evaluated by introducing variables $s_{1}, s_{1}^{\prime}, \ldots, s_{2}, s_{2}^{\prime}, \ldots$ in a way analogous to above. Namely, a first index $i_{1}$ (analogous to $\left.\Delta_{1}\right)$ is chosen and all variables $\varepsilon_{i, j}$ are replaced by $\varepsilon_{i, j}\left(s_{1}\right)$, where:

$$
\varepsilon_{i, j}\left(s_{1}\right)=\varepsilon_{i, j}\left[s_{1}+\left(1-s_{1}\right)\left[\left(\delta_{i_{1}, i} \delta_{i_{1}, j}+\left(1-\delta_{i_{1}, i}\right)\left(1-\delta_{i_{1}, j}\right)\right]\right],\right.
$$


where $i, j$ play the role of $x, y$ in $C(x, y)$ or $C\left(x, y ; s_{1}\right)$. We then consider the $1^{\text {st }}$ order Taylor expansion $F \equiv F(1)=F(0)+\int_{0}^{1} \frac{d}{d s_{1}} F\left(s_{1}\right)$, where $F(0)$ factorizes, and so forth,
with:

$$
\begin{aligned}
\varepsilon_{i j}\left(\bar{s}, s_{k}^{(q)}\right)= & \varepsilon_{i j}(\bar{s})\left[s_{k}^{(q)}+\left(1-s_{k}^{(q)}\right)\left\{\delta\left(i, i_{1}, \ldots, i_{k}\right) \delta\left(j ; i_{1}, \ldots, i_{k}\right)\right.\right. \\
& \left.+\left(1-\delta\left(i ; i_{1}, \ldots, i_{k}\right)\right)\left(1-\delta\left(j ; i_{1}, \ldots, i_{k}\right)\right)\right\},
\end{aligned}
$$

where $\bar{s}=\left(s_{1}^{\left(r_{1}-1\right)}, s_{2}^{\left(r_{2}-1\right)}, \ldots, s_{k-1}^{\left(r_{k-1}-1\right)}\right)$ and $\delta\left(i ; i_{1}, \ldots, i_{k}\right)=1$ if $i$ is equal to one of the indices $i_{1}, \ldots, i_{k}$ and to zero otherwise (the indices $i_{1}, \ldots, i_{k}$ play the roles of $\left.\Delta_{1}, \ldots, \Delta_{k}\right)$. This procedure provides an expansion of $F(\varepsilon)$, or its connected part, of the form:

$$
\begin{aligned}
F(\varepsilon)= & \sum_{G, P} \int d s M(s ; G, P)\left(\prod_{i, j \in G} \varepsilon_{i j}^{n_{i j}, \jmath}\right) \\
& \times\left(\prod_{i, j} n_{i j} !+\sum_{\substack{\left\{n_{k}^{\prime}, l\right\} \\
\sum n_{k, l}^{\prime} \geqq 1}} C_{\left\{n_{k, l}^{\prime}\right\}} \prod_{k, l} \varepsilon_{k, l}(s)^{n_{k, l}^{\prime}}\right),
\end{aligned}
$$

where the sum runs over (admissible) connected graphs $G$ in the same class as before and where, moreover, $C_{\left\{n_{k, l}^{\prime}\right\}} \neq 0$ only if there is no procedure $P$ giving rise to the graph $\left\{n^{\prime \prime}\right\}=\{n\}+\left\{n^{\prime}\right\}$. In fact, if $n_{k, l}^{\prime} \neq 0$, then the variable $s$ corresponding to a possible coupling between $k$ and $l$ is not fixed at zero. Hence, the expansion relative to this $s$ has been stopped and $\left\{n^{\prime \prime}\right\}$ cannot be obtained by a procedure $P$. Equation (32) follows by identifying the coefficients of $\prod_{(i, j) \in G} \varepsilon_{i j}^{n_{i}, j}$ in (33) and (36). Q.E.D.

In view of this lemma, we now state the following bound.

Proposition 2. a) If there is no $z_{r} \in X, r=1, \ldots, l, \exists$ constants $C_{1}(\varepsilon), C_{2}$ independent of $X, G$ (and $\Lambda$ ) such that ( for any $\varepsilon>0$ ):

$$
|I(X, G)|<\left[\prod_{l \in G}|\lambda|^{1 / 2} C_{1}(\varepsilon) e^{-(m-\varepsilon) d(l)}\right] C_{2}^{|X|},
$$

where $d(l)$ is the distance of the centers of the squares of $X$ joined by line $l$.

b) If there are $l^{\prime}$ points $z_{r}$ in $X, \exists C_{1}(\varepsilon), C_{2}$ (independent of $X, G, \Lambda$ ) such that:

$$
\begin{aligned}
|I(X, G)|< & \left(1+\left|\ln \operatorname{Inf}_{\substack{i, j, i \neq j \\
\text { such that } \\
z_{i} \in X, z_{J} \in X}}\right| z_{i}-z_{j} \mid\right)^{6}|\lambda|^{\operatorname{Sup}\left(0, \frac{n(G)}{2}-3\right)+l^{\prime}} \\
& \times\left[\prod_{l \in G} C_{1}(\varepsilon) e^{-(m-\varepsilon) d(l)}\right] C_{2}^{|X|},
\end{aligned}
$$

where $n(G)$ is the number of lines in $G$.

Proof. In view of (32), it is sufficient to bound $|J(X, G, P, s)|$. Uniform bounds on the latter can be obtained by an adaptation of usual methods (see [20] and references therein). The exponential fall-off factors $e^{-(m-\varepsilon) d(l)}$ arise from the decrease of the propagators $C(u, v)$ as $e^{-m|u-v|} \leqq \operatorname{cst} e^{-m d(l)}$. A fall-off factor $e^{-\varepsilon|u-v|}$ is kept to control the sum over the terms generated by the derivatives $\frac{\delta}{\delta \varphi}$ and, for each of these terms, the sum over the contraction schemes of the fields. The independence of the bound on $P$ and $s$ arises from the inequality $0 \leqq C(x, y ; s) \leqq C(x, y)$, which 
gives directly the inequality $\int \pi \varphi d \mu(s) \leqq \int \pi \varphi d \mu(1)$ and can be shown to yield also the inequality $\int e^{-\lambda \int_{X}: \varphi^{4}:(x) d x} d \mu(s) \leqq \operatorname{cste}^{|X|}$, with a constant independent of $s$. The factor $\lambda^{1 / 2}$ in (37) for each line $l$ arises from the fact that there are four fields by vertex and that propagators are obtained by contraction of two fields. The power of $\lambda$ in (38) is different because some propagators can be obtained by contraction of fields $\varphi\left(z_{r}\right)$. On the other hand, a propagator obtained by contraction of fields $\varphi\left(z_{r}\right), \varphi\left(z_{r^{\prime}}\right)$ behaves at short distances like $\ln \left|z_{r}-z_{r^{\prime}}\right|$. The first factor in the righthand side of (38) accounts for this fact.

We conclude this section with the following comment. If $|X|$ is composed of only one square $\Delta$, there is only one term $I(X, G, P) \equiv I(\Delta)$ equal to:

$$
I(\Delta)=\int \prod_{i \text { such that } z_{i} \in \Delta}\left(\lambda: \varphi^{n_{i}}:\left(z_{i}\right)\right) e^{-\lambda \int: \varphi^{4}(x): d x} d \mu(\varphi) .
$$

$I(\Delta)$ is a constant $a(\neq 0$ : see [21]) independent of $\Delta$, if $\Delta$ contains no point $z$. Equation (7) follows.

\section{4. $p^{\text {th }}$-Order Expansions of Connected Functions}

\subsection{Description}

While the Mayer procedure might be applied in a more simple form for the purposes of this section (introduction of non-overlap factors $1+\chi$ between subsets $X_{i}$ rather than between squares), we present it in a form that is also convenient for the study of irreducible kernels. Let $\Delta_{0,1}, \ldots, \Delta_{0, \sigma}$ denote the different squares of $A$ containing one or more points $z_{r}, r=1, \ldots, \sigma(\sigma \geqq 1)$. Equation (7) can be written:

$$
\begin{aligned}
& I_{\Lambda}\left(z_{1}, \ldots, z_{l}\right)=a^{|\Lambda|} \sum_{N \leqq|\Lambda|-\sigma} \frac{a^{-(N+\sigma)}}{N !} \\
& \times \sum_{\substack{\Delta_{1}, \ldots, \Delta_{N} \\
\Delta_{i} \subset \Lambda, i=1, \ldots, N}} \prod_{\substack{(\alpha, \beta), \alpha<\beta \\
\alpha, \beta=(0,1), \ldots,(0, \sigma), 1, \ldots, N}}\left(1+\chi\left(\Delta_{\alpha}, \Delta_{\beta}\right)\right) \\
& \times \sum_{\substack{\text { partitions } \omega_{1}, \ldots, \omega_{q} \\
\text { of }(0,1), \ldots,(0, \sigma), 1, \ldots, N ; \\
q \geqq 1}}^{\sum_{G_{1}, \ldots, G_{q}}} \prod_{j=1}^{q} I\left(X_{j}, G_{j}\right),
\end{aligned}
$$

where $\chi\left(\Delta_{\alpha}, \Delta_{\beta}\right)=-1$ (i.e. $1+\chi=0$ ) if $\Delta_{\alpha}=\Delta_{\beta}, \chi=0$ (i.e. $1+\chi=1$ ) if $\Delta_{\alpha} \neq \Delta_{\beta}$, the factors $1+\chi$ account for the fact that $\Delta_{1}, \ldots, \Delta_{N}$ have to be different from each other and from $\Delta_{0,1}, \ldots, \Delta_{0, \sigma}$, and the sum $\sum^{\prime}$ runs over partitions including no subset $\omega_{j}$ of one element among $1, \ldots, N$. The factor $(N !)^{-1}$ accounts for the fact that each configuration of given subsets $X_{j}$ is obtained $N$ ! times when $\Delta_{1}, \ldots, \Delta_{N}$ vary in $\Lambda$. The graphs $G_{j}$ are connected graphs between the indices of $\omega_{j}$ with the same properties as in Sect. 3 and $X_{j}=\{\Delta\}_{\gamma \in \omega_{j}}$. Factors $1+\chi\left(\Delta_{\alpha}, \Delta_{\beta}\right), \alpha, \beta=(0,1), \ldots,(0, \sigma)$ are equal to one and can thus be removed.

Through expansions of the products $1+\chi$, one obtains in turn an expression of the form:

$$
I_{\Lambda}\left(z_{1}, \ldots, z_{l}\right)=a^{|\Lambda|} \sum_{N \leqq|\Lambda|-\sigma} \frac{1}{N !} \sum_{\substack{\Delta_{1}, \ldots, \Delta_{N} \\ \Delta_{i} \subset \Lambda, i=1, \ldots, N}} \sum_{\substack{\omega_{1}, \ldots, \omega_{q} \\ q \geqq 1}} \sum_{\mathbf{G}_{1}, \ldots, \mathbf{G}_{q}} \prod_{j=1}^{q} \mathbf{I}\left(X_{j}, \mathbf{G}_{j}\right) .
$$


In (41), the graph $\mathbf{G}_{j}$ (see example in Fig. 4) include lines associated with propagators (as before) and Mayer lines associated with factors $\chi$. They are connected when both types of lines are taken into account. The factors $I$ are defined as products of terms $a^{|X|} I$ associated with subgraphs already connected by lines - and of factors $\chi$ associated with Mayer lines joining indices $\alpha, \beta(\chi=0$ if $\Delta_{\alpha} \neq \Delta_{\beta}, \chi=-1$ if $\left.\Delta_{\alpha}=\Delta_{\beta}\right)$. The value of $I(X, G)$ when some squares of $X$ coincide is arbitrary. In this subsection, it can be taken equal to zero, but a different choice is made in Sect. 5, namely: $I=0$ whenever two squares that belong to a common $(p-1)$-irreducible subgraph coincide, otherwise $I$ is defined by a direct extension of factorization properties.

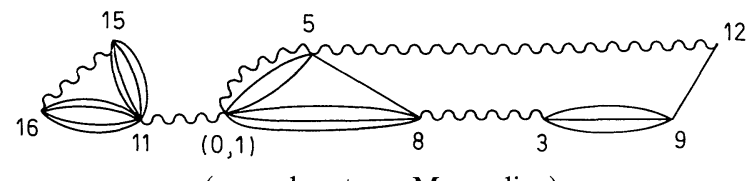

Fig. 4 A graph $\mathbf{G}(p=4, \omega=\{(0,1), 3,5,8,9,11,12,15,16\})$

Finally, the separation of those $\omega_{j}$ that contain one or more of the indices $(0,1), \ldots,(0, \sigma)$, and of remaining ones, leads to a partition of the set of $N$ indices into subsets of $N_{1}$ and $N_{2}$ indices $\left(N_{1}+N_{2}=N\right)$, which can be chosen to be the indices $1, \ldots, N_{1}$ and $N_{1}+1, \ldots, N_{2}$, if a multiplication factor $N !\left(N_{1} ! N_{2} !\right)^{-1}$ is added. This leads to a factorization of $I_{A}$ into the term $Z_{A}$ (no variable $z_{r}, \sigma=0$ ) and a term which is equal to $H_{\Lambda}$ in view of (16). (Values of $N_{1}, N_{2}, N_{1} \leqq|\Lambda|-\sigma, N_{2} \leqq|\Lambda|-\sigma$, such that $N=N_{1}+N_{2}>|\Lambda|-\sigma$ are zero in view of the product $\prod_{(\alpha, \beta)}$ in (40) and can thus be added to $\left.I_{\Lambda} \cdot\right)$ Hence:

$$
H_{\Lambda}\left(z_{1}, \ldots, z_{l}\right)=\sum_{N \leqq|\Lambda|-\sigma} \frac{1}{N !} \sum_{\Delta_{1}, \ldots, \Delta_{N}} \sum_{\omega_{1}, \ldots, \omega_{q}}^{\prime \prime} \sum_{\mathbf{G}_{1}, \ldots, \mathbf{G}_{q}} \prod_{j=1}^{q} \mathbf{I}\left(X_{j}, \mathbf{G}_{j}\right),
$$

where the sum $\sum^{\prime \prime}$ runs over partitions $\omega_{1}, \ldots, \omega_{q}$ such that each $\omega_{j}$ contains one or more of the indices $(0,1), \ldots,(0, \sigma)$. Thus, $H_{A}$ can be written in the form:

$$
H_{\Lambda}\left(z_{1}, \ldots, z_{l}\right)=\sum_{\substack{\text { partitions } \\ \pi_{1}, \ldots, \pi_{k} \\ \text { of }(1, \ldots, \sigma), k \geqq 1}} \prod_{i=1}^{k}\left[\sum_{N \leqq|\Lambda|-\left|\pi_{i}\right|} \frac{1}{N !} \sum_{\Delta_{1}, \ldots, \Delta_{N}} \sum_{\mathbf{G}_{i}} \mathbf{I}\left(X_{i}, \mathbf{G}_{i}\right)\right],
$$

where the last sum runs over connected graphs $\mathbf{G}_{i}$ between the indices $(0, r)$ that belong to $\pi_{i}$ and $1, \ldots, N$. If the variables $z_{1}, \ldots, z_{l}$ all belong to different squares, i.e. $\sigma=l$, the standard definition of connected functions (which expresses $H_{A}$ as a sum over partitions of $z_{1}, \ldots, z_{l}$ of products of functions $H_{A}^{c}$ ) yields in turn the expansion (9) of $H_{\Lambda}^{c}$ :

$$
H_{\Lambda}^{c}\left(z_{1}, \ldots, z_{l}\right)=\sum_{N \leqq|\Lambda|-l} \frac{1}{N !} \sum_{\Delta_{1}, \ldots, \Delta_{N}} \sum_{\mathbf{G}} \mathbf{I}(X, \mathbf{G}),
$$

where $X$ is the set of squares $\Delta_{0,1}, \ldots, \Delta_{0, l}, \Delta_{1}, \ldots, \Delta_{N}$. 
If $\sigma<1$, one may start from the expression (42) and add to its right-hand side the term $H_{A}^{\prime}$ defined below and in fact equal to zero. Let $\pi$ be the partition of indices $1, \ldots, l$ (or of $z_{1}, \ldots, z_{l}$ ) into those $\sigma$ subsets such that the variables $z_{i}$ of each subset belong to a common square $\Delta_{0, r}$. We then consider non-trivial subpartitions of $\pi$ and, whenever the induced partition of indices for a given $r$ has $\gamma(r)>1$ elements, replace $\Delta_{0, r}$ by $\gamma(r)$ coincident squares $\Delta_{0, r, 1}, \Delta_{0, r, 2}, \ldots H_{A}^{\prime}$ is then the sum, over all non-trivial subpartitions of $\pi$, of terms analogous to the right-hand side of (42), except that $\Sigma^{\prime \prime}$ is replaced by a sum over partitions $\omega_{1}, \ldots, \omega_{q}$ of the indices $(0,1,1)$, $(0,1,2), \ldots,(0, \sigma, 1),(0, \sigma, 2), \ldots, 1, \ldots, N$ such that each $\omega_{j}$ contains one or more of the indices $(0,1,1), \ldots,(0, \sigma, \gamma(\sigma))$.

The fact that $H_{A}^{\prime} \equiv 0$ is due to the coincidence of at least two squares $\Delta_{0, r, \alpha}$, as can be checked easily. The definition of connected functions then gives:

$$
H_{\Lambda}^{c}\left(z_{1}, \ldots, z_{l}\right)=\sum_{\text {subpartitions of } \pi} \sum_{N \leqq|\Lambda|-\sigma} \frac{1}{N !} \sum_{\Delta_{1}, \ldots, \Delta_{N}} \sum_{\mathbf{G}} I(X, \mathbf{G}),
$$

where the graphs $\mathbf{G}$ are here connected graphs in the same sense as above, but (for each given subpartition of $\pi$ ) between the indices $(0,1,1), \ldots,(0, \sigma, \gamma(\sigma)), 1, \ldots, N$. The first sum $\sum$ includes the trivial subpartition, i.e. $\pi$ itself. While $H_{A}^{\prime} \equiv 0$, the various contributions to the right-hand side of (44), which involve only connected graphs, are not zero in general.

\subsection{Convergence and Decay in the $\Lambda \rightarrow \infty$ Limit}

We now give a proof of Proposition 1. For simplicity, we consider only the case when $z_{1}, \ldots, z_{l}$ belong to different squares. The treatment of Eq. (44) is similar. As already mentioned, we use a $p^{\text {th }}$-order expansion, $p \geqq 1$, in view of the adaptation in Sect. 5, even though the $p=1$ expansion is sufficient for present purposes.

Being given a connected graph $\mathbf{G}$, let $G_{1}, G_{2}, \ldots$ be its subgraphs already connected by lines - It is convenient in this section to regroup together all terms associated with graphs $\mathbf{G}^{\prime}$ that differ only by their sets of Mayer lines inside each subgraph $G_{i}$. The summation over $\Delta_{1}, \ldots, \Delta_{N}$ is then restricted as originally by the non-overlap conditions $\Delta_{v} \neq \Delta_{v}$, inside each $G_{i}$, and it is sufficient to consider graphs $\mathbf{G}$ with Mayer lines joining vertices of different subgraphs. Moreover, in view of the previous non-overlap conditions, the only $\mathbf{G}$ that give non-zero contributions are those that have at most one Mayer line between any vertex of a subgraph $G_{i}$ and any subgraph $G_{j}, j \neq i$. A skeleton graph $\hat{\mathbf{G}}$ is then associated to each graph $\mathbf{G}$ as follows. Starting from the subgraph, denoted $G_{1}$, that contains e.g. the index $(0,1)$, we consider all subgraphs $G_{i}, i \neq 1$, of a "first shell," namely those linked to $G_{1}$ by at least one Mayer line. If there are several Mayer lines between $G_{1}$ and $G_{i}$, all are removed, except that attached to the vertex of lowest index in $G_{1}$; the order of vertices of $G_{1}$ is their natural order in the sequence $(0,1), \ldots,(0,1), 1, \ldots, N$ : in the example of Fig. 5

Fig. 5

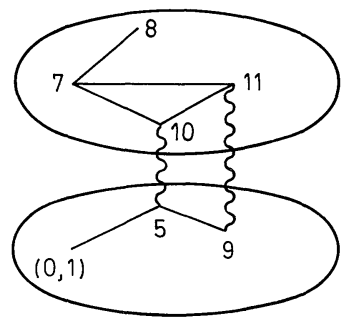


the Mayer line joining the indices 9,11 is removed (and the line joining 5, 10 is kept). All Mayer lines between the subgraphs $G_{i}$ of the first shell are also removed. Next, one considers all subgraphs of a "second shell," namely those attached to subgraphs of the first shell by Mayer lines. The same procedure as above is applied (using e.g. the natural order of all vertices of all subgraphs of the first shell), and so forth. The skeleton graph $\hat{\mathbf{G}}$ obtained is of the form shown in Fig. 6:

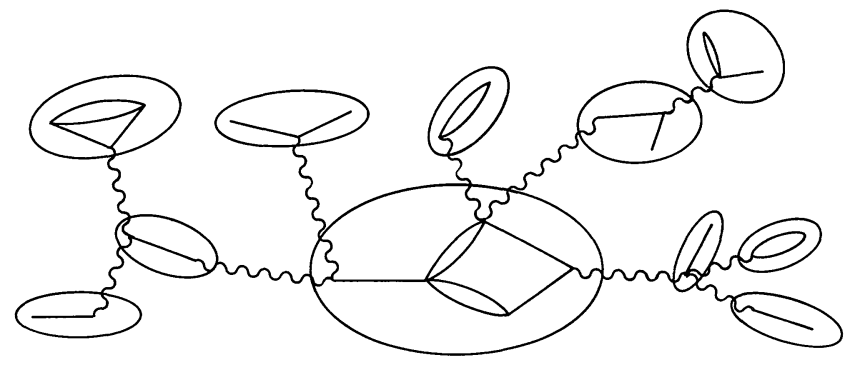

Fig. 6. A skeleton graph

From each vertex of a given subgraph, zero, one or more lines are issued, each one joining this subgraph to one subgraph of the next shell.

The summation in (44) over all graphs $\mathbf{G}$ that have the same skeleton $\hat{\mathbf{G}}$ amounts to introduce non-overlap functions between squares that belong to different subgraphs of the same shell and some squares that belong to subgraphs of successive shells. The uniform bounds (17) of Proposition 1 then follow from uniform bounds in modulus on all contributions corresponding to different skeleton graphs, non-overlap functions, as also the factors -1 of each Mayer line, being bounded in modulus by 1 . We first remark, as mentioned in Sect. 1, that a common uniform fall-off factor $e^{-\left(m-\varepsilon^{\prime}\right) l_{0}\left(z_{1}, \ldots, z_{l}\right)}, \varepsilon^{\prime} \geqq \varepsilon$ can be extracted for all terms by extracting a factor $e^{-\left(m-\varepsilon^{\prime}\right) d(l)}$ from each factor $e^{-(m-\varepsilon) d(l)}$ of the bounds (37), (38). If $\varepsilon^{\prime}$ is chosen $>\varepsilon$, we still have at our disposal a factor $e^{-\left(\varepsilon^{\prime}-\varepsilon\right) d(l)}$ for each line.

For any given $N$, skeleton graphs can be specified by indicating the set of indices of the first subgraph $G_{1}$, then for each index a set of zero, one or more Mayer lines that are issued from it and the set of indices of corresponding subgraphs, and so forth. (Subgraphs $G_{1}, G_{2}, \ldots$ have also to be specified.) The choice of $N_{1}, N_{2}, \ldots$ indices among $1, \ldots, N$ for each subset amounts to replace the factor $(N !)^{-1}$ in the bounds by $\prod_{i}\left(N_{i} !\right)^{-1}$. The choice of the index in each subset by which this subset is attached to a preceding one and the specification of possible external indices $(0,2), \ldots,(0, l)$ contained in each subset can be taken into account, e.g. by including a factor $k N_{i}$, where $k$ is a given (fixed) constant, for each subset. Finally, uniform bounds independent of $\Lambda$ will be obtained by removing the conditions $N \leqq|\Lambda|-l$ and $\Delta_{i} \in \Lambda$, and considering independent sums over $N_{1}>0$, $N_{2}>0, \ldots$. The following bound will be used (in successive steps) for each subset, the index 0 , denoting either an external index $(0, \sigma)$ or the index by which the subset considered is attached. (Possible subsets with one external, and no internal, index are treated separately):

$$
\sum_{N \geqq 1} \frac{C^{N}}{N !} \sum_{\substack{\Delta_{1}, \ldots, \Delta_{N} \\ \Delta_{i} \cap \Delta_{j}=\emptyset, i \neq j}} \sum_{G(0,1, \ldots, N)} \prod_{l \in G}\left(C^{\prime} e^{-\eta d(l)}\right) \leqq C^{\prime \prime}
$$


for sufficiently small $C^{\prime}$ (a condition that will be fulfilled in the application for sufficiently small $\lambda$ ), with $C^{\prime \prime}$ arbitrarily small if $C^{\prime}$ is sufficiently small.

Proof of (46). The bound (46) can be obtained, e.g. by bounding the sum over relevant graphs $G$ by a sum over all trees joining $0,1, \ldots, N$ and for each tree a sum over all graphs obtained by adding, for each vertex $v$, sets of $0,1, \ldots, p$ lines from $v$ to each other vertex.

The sum, with a vector $C^{\prime} e^{-\eta d(l)}$ for each line, over all possible sets of $\leqq p$ lines joining a given square $\Delta_{v}$ to all other squares in $R^{2}$ is a finite constant $K$ if $C^{\prime}$ is sufficiently small.

For each tree $T, \sum_{\Delta_{1}, \ldots, \Delta_{N}} \prod_{l \in T}\left(C^{\prime} e^{-\eta d(l)}\right)$ is bounded by $C^{\prime N}\left[\sum_{\Delta \neq \Delta_{0}} e^{-\eta d(l)}\right]^{N}$. Finally, the number of trees $T(0, \ldots, N)$ is bounded by $\operatorname{Cste}^{N} N$ ! The bound (46) follows. Q.E.D.

The bound (46) is then applied, starting from subsets in the most remote shell up to the first shell. The fact that an arbitrary number $(\geqq 0)$ of subsets are attached (by Mayer lines) to each vertex of a subgraph leads to add a (fixed) multiplicative constant, for each vertex, in the bounds [equal to $\left(1-C^{\prime \prime}\right)^{-1}$ if we leave aside the possibility that some subsets contain external vertices. This possibility is taken into account by modifying this constant]. Hence a bound independent of the number of successive shells is obtained if this constant has been included in the constant $C$ of Eq. (46) already at the first stage. Q.E.D.

The fact that $H_{A}^{c}$ converges to a well defined function $H^{c}$ in the $A \rightarrow \infty$ limit is proved by showing in a way similar to above that $\left|H_{A}^{c}-H_{A^{\prime}}^{c}\right|$ is uniformly bounded by $c(\Lambda), c(\Lambda) \rightarrow 0$ as $\Lambda \rightarrow \infty, \forall \Lambda^{\prime} \supset \Lambda$ : in fact, exponential fall-off factors with the distance between the set of points $z_{1}, \ldots, z_{l}$ and the boundary of $\Lambda$ arise since $H_{A}^{c}-H_{A^{\prime}}^{c}$ is a sum over connected graphs and over squares $\Delta_{1}, \ldots, \Delta_{N}$ one of which at least belongs to $\Lambda^{\prime} \backslash \Lambda$. The independence of $H^{c}$ on the way the limit is obtained (i.e. on the sequence of boxes $\Lambda$ ) can also be established similarly.

\section{The Bethe-Salpeter Kernel: 4 Particle Decay}

For the purposes of this section, we consider a cluster expansion of order $p=4$. A $3^{\text {rd }}$-order expansion would be sufficient to get momentum space analyticity of the Bethe-Salpeter kernel $G$ arbitrarily close to the 3-particle threshold (for sufficiently small $\lambda$ ). The use of a $4^{\text {th }}$-order expansion will provide analyticity arbitrarily close to the 4-particle threshold, as expected in an even theory.

The connected 4-point function $S_{\Lambda}^{c}\left(x_{1}, \ldots, x_{4}\right)$ can be written, in view of (14), (16) in the form:

$$
\begin{aligned}
S_{\Lambda}^{c}\left(x_{1}, \ldots, x_{4}\right)= & \int_{\substack{z_{i} \in \Lambda \\
i=1, \ldots, 4}} d z_{1} \ldots d z_{4} \prod_{i=1}^{4} C\left(x_{i}-z_{i}\right)\left[\lambda \delta\left(z_{1}-z_{2}\right) \delta\left(z_{1}-z_{3}\right) \delta\left(z_{1}-z_{4}\right)\right. \\
& +\delta\left(z_{1}-z_{2}\right) \delta\left(z_{1}-z_{3}\right) H_{\Lambda, 1}^{c}\left(z_{1}, z_{4}\right)+\ldots \\
& +\delta\left(z_{1}-z_{2}\right) \delta\left(z_{3}-z_{4}\right) H_{\Lambda, 2}^{c}\left(z_{1}, z_{3}\right)+\ldots \\
& \left.+\delta\left(z_{1}-z_{2}\right) H_{\Lambda}^{c}\left(z_{1} ; z_{3} ; z_{4}\right)+\ldots+H_{\Lambda}^{c}\left(z_{1}, z_{2}, z_{3}, z_{4}\right)\right]
\end{aligned}
$$


A corresponding expansion of $S_{\Lambda}^{c}$ follows from those of the functions $H_{\Lambda}^{c}$. It gives, by direct graphical inspection:

$$
S_{\Lambda}^{c}\left(x_{1}, \ldots, x_{4}\right)=\int_{\substack{u_{i} \in \Lambda \\ i=1, \ldots, 4}}\left[\prod_{i=1}^{4} S_{\Lambda}\left(x_{i}, u_{i}\right)\right] F_{\Lambda}\left(u_{1}, \ldots, u_{4}\right) d u_{1} \ldots d u_{4},
$$

where $S_{\Lambda}\left(x_{1}, x_{2}\right)$ is the 2-point function and $F_{\Lambda}$, the amputated, connected 4-point function, admits an expansion analogous to that of $S_{\Lambda}^{c}$ except that it is restricted to graphs $\mathbf{G}$ that are one-particle irreducible in all $1 \rightarrow 3$ channels of the form $(i ; j, k, l)$. We then define $G_{\Lambda}\left(u_{1}, \ldots, u_{4}\right)$ as the sum over all graphs that are moreover 2particle irreducible in the channel $(1,2 ; 3,4)$. We note that, in the definition of irreducibility used here, Mayer lines cannot be cut.

The one-particle irreducible 2-point function $K\left(u_{1}, u_{2}\right)$ is defined similarly as follows. The 2-point function can be written:

$$
\begin{aligned}
S_{\Lambda}\left(x_{1}, x_{2}\right) & =\int_{\substack{u_{i} \in \Lambda \\
i=1,2}} d u_{1} d u_{2} \prod_{i=1,2} C\left(x_{i}, u_{i}\right)\left[\lambda \delta\left(u_{1}-u_{2}\right)\left\langle: \varphi^{2}:\right\rangle_{\Lambda}+H_{\Lambda}^{\prime c}\left(u_{1}, u_{2}\right)\right] \\
& =\int_{u_{i} \subset \Lambda} d u_{1} d u_{2} \prod_{i=1,2} C\left(u_{i}, x_{i}\right) \hat{S}_{\Lambda}\left(u_{1}, u_{2}\right) .
\end{aligned}
$$

Then, $K_{\Lambda}\left(u_{1}, u_{2}\right)$ is the sum over all 1-particle irreducible graphs in the expansion of $\hat{S}_{\Lambda}\left(u_{1}, u_{2}\right)$.

The following equations hold by direct inspection:

$$
\begin{gathered}
S_{\Lambda}=C+C K_{\Lambda} S_{\Lambda}, \\
F_{\Lambda}=G_{\Lambda}+G_{\Lambda^{\circ}{ }_{A}} F_{\Lambda},
\end{gathered}
$$

where $C K_{\Lambda} S_{\Lambda}\left(x_{1}, x_{2}\right)$ is the double convolution integral

$$
\int_{u_{1}, u_{2} \in \Lambda} C\left(x_{1}, u_{1}\right) K_{\Lambda}\left(u_{1}, u_{2}\right) S_{\Lambda}\left(u_{2}, x_{2}\right) d u_{1} d u_{2}
$$

and $G_{A^{\circ}(\Lambda)} F_{A}$ is a Feynman-type convolution integral with 2-point functions on internal lines:

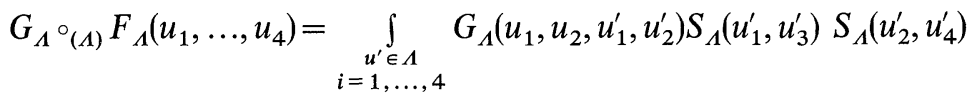

$$
\begin{aligned}
& \times F_{\Lambda}\left(u_{3}^{\prime}, u_{4}^{\prime}, u_{3}, u_{4}\right) \prod_{i=1}^{4} d u_{i}^{\prime},
\end{aligned}
$$

i.e. Eq. (51) is the usual Bethe-Salpeter equation apart from the dependence in $\Lambda$. In view of (47), (49), $K_{\Lambda}$ and $G_{A}$ admit decompositions of the form:

$$
\begin{gathered}
K_{\Lambda}\left(u_{1}, u_{2}\right)=\lambda \delta\left(u_{1}-u_{2}\right)\left\langle: \varphi^{2}:\right\rangle_{\Lambda}+\lambda^{2} K_{\Lambda}^{\prime}\left(u_{1}, u_{2}\right), \\
G_{\Lambda}\left(u_{1}, \ldots, u_{4}\right)=\lambda \delta\left(z_{1}-z_{2}\right) \delta\left(z_{1}-z_{3}\right) \delta\left(z_{1}-z_{4}\right)+\ldots+G_{\Lambda}^{\prime}\left(u_{1}, \ldots, u_{4}\right) .
\end{gathered}
$$

The following result is proved below:

Proposition 3. $\forall \varepsilon>0, \exists \lambda_{\varepsilon}, C_{\varepsilon}$ (independent of $\Lambda$ ) such that $K_{\Lambda}^{\prime}$ and the functions $G_{\Lambda}^{\prime}$ converge to well defined functions in the $\Lambda \rightarrow \infty$ limit, $\forall \lambda$ such that $|\lambda|<\lambda_{\varepsilon}$. Moreover, the following bounds hold $\left(\forall \Lambda\right.$ finite or infinite), $\forall \lambda,|\lambda|<\lambda_{\varepsilon}$ :

$$
\begin{gathered}
\left|K_{\Lambda}^{\prime}\left(u_{1}, u_{2}\right)\right|<C_{\varepsilon}|\lambda|\left(1+|\ln | u_{1}-u_{2}||\right)^{3} e^{-3(m-\varepsilon)\left|u_{1}-u_{2}\right|}, \\
\left|G_{\Lambda}^{\prime}\left(u_{1}, \ldots, u_{l}\right)\right|<C_{\varepsilon}|\lambda|\left(1+|\ln \operatorname{Inf}| u_{i}-u_{j}||\right)^{6} e^{-(m-\varepsilon) l_{3}\left(u_{1}, u_{2} ; u_{3}, u_{4}\right)},
\end{gathered}
$$


where $l_{3}$ is the shortest length of all connected graphs joining $u_{1}, \ldots, u_{4}$ and possibly intermediate points, and 3-particle irreducible in the channel $(1,2 ; 3,4)$ as also 2particle irreducible in $1 \rightarrow 3$ channels.

Finally, $C K_{\Lambda} S_{\Lambda}$, respectively $G_{\Lambda}{ }^{\circ}{ }_{(\Lambda)} F_{\Lambda}$, converges in the $\Lambda \rightarrow \infty$ limit to $C K S$, respectively $G \circ F$, and $F$ and $G$ satisfy the Bethe-Salpeter equation $F=G+G \circ F$.

Proof. We prove below the bound (56) on $G_{\Lambda}^{\prime}\left(u_{1}, \ldots, u_{4}\right)$. Other bounds are proved similarly. The method is analogous to that used in Sect. 4.2 with, however, some difference arising from a different definition of skeleton graphs: the set of graphs $\mathbf{G}$ having a graph $\hat{\mathbf{G}}$ as a skeleton graph (in the sense of Sect. 4.2) contains in fact graphs that do not belong to the expansion of $G_{A}^{\prime}$. Being given a graph $\mathbf{G}$, its skeleton graph $\hat{\mathbf{G}}$ will now be defined as follows. We first consider $\hat{\mathbf{G}}$. It no longer satisfies in general the irreducibility properties of the graphs in the expansion of $G_{A}^{\prime}$ and has in fact a structure of the form:

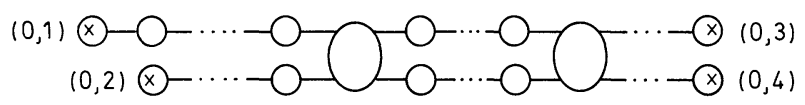

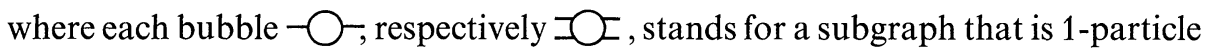
irreducible, respectively 2-particle irreducible, in the channels determined by the incoming and outgoing lines, and has the same form (no loop arising from the Mayer lines) as before. [The vertex $(0,1)$ is now replaced by one of the incoming vertices.]

$\hat{\hat{G}}$ is defined by keeping a further "minimal" set of Mayer lines (that do not belong to $\hat{\mathbf{G}})$. Starting from the external bubble $b_{1}$ on the left containing, e.g. the vertex $(0,1)$, we consider the set of all Mayer lines of $\mathbf{G}$ that join $b_{1}$ to other bubbles. This defines a 2-particle irreducible set $B_{1}$ that contains $b_{1} . B_{1}$ is composed of all bubbles "between" extremal bubbles of the latter set. (A bubble $b$ of a set is extremal on the right, respectively on the left, if there is no other bubble $b^{\prime}$ in this set on the right, respectively on the left, of $b$. A bubble $b$ is between $b^{\prime}, b^{\prime \prime}$ if $b^{\prime}$ is on its left and $b^{\prime \prime}$ on its right or vice versa. The relation left-right is well defined in the natural way for any pair of bubbles, except bubbles - - that belong to different lines - -..- - and have no bubble $工 \mathrm{O}$ between them: such bubbles have no relation left-right.) We then keep in $\hat{\mathbf{G}} 1,2$ or 3 Mayer lines that join $b_{1}$ to the 1,2 or 3 other extremal bubbles of $B_{1}$. If there are several Mayer lines joining $b_{1}$ to another extremal bubble $b_{1}^{\prime}$, we keep more precisely e.g. the line, among those issued from the vertex of lowest order in $b_{1}$, joining the vertex of lowest order in $b_{1}^{\prime}$.

The same procedure is now applied to $B_{1}$ and so forth. We note that Mayer lines issued from $B_{1}$ cannot be issued from $b_{1}$. More generally, Mayer lines issued from $B_{i}$ to $B_{i+1}$ cannot be issued from $B_{i-1}$.

As before, the resummation of all graphs $\mathbf{G}$ that have the same skeleton graph $\hat{\mathbf{G}}$ gives non-overlap conditions, to be bounded by 1 in bounds on absolute values (and skeleton graphs that differ only by the labelling of vertices lead in turn to the same bounds). A bound on the sum over all possible structures of graphs $\mathbf{G}$, and for each bubble over all possible (2-particle irreducible) substructures can be obtained e.g. as follows. (More refined estimates are possible if one is interested in obtaining 
a better radius of convergence in $\lambda$, e.g. by regrouping graphs $\hat{\mathbf{G}}$ that differ by the number of intermediate bubbles in the successive subsets $B_{1}, B_{2}, \ldots$.) First, for any $\hat{\mathbf{G}}$, the number of graphs $\hat{\mathbf{G}}$ is bounded by $8^{N}$, where $N$ is the total number of squares of $\mathbf{G}$, in view of the previous construction. After extracting a common fall off factor $e^{-(m-\varepsilon) l_{3}\left(u_{1}, \ldots, u_{4}\right)}$ we remove in the bounds the coincidence relations due to the Mayer lines in $\hat{\mathbf{G}} / \hat{\mathbf{G}}$ and are then led to prove a bound on the sum over structures of graphs $\hat{\mathbf{G}}$, with a further multiplicative factor 8 now associated to each vertex. (For sufficiently small $\lambda$, this factor does not modify the argument.)

For each bubble $b$, the set of possible substructures is a subset of those occurring in Sect. 4.2. Hence bounds that include (i) a remaining exponential falloff factor between points from which external line of $b$ are issued and (ii) an arbitrary small factor for $\lambda$ sufficiently small (except possibly for a finite, fixed, number of cases), are obtained in the same way as in Sect. 4.2. The exponential falloff factors associated with the bubbles together with those associated with the explicit lines — between bubbles allow one in turn to get a uniform bound of the form $\operatorname{cste} C^{n}$, where $n$ is the number of bubbles and $C$ is arbitrarily small (for $\lambda$ sufficiently small). The bound (56) follows.

The fact that the functions $F_{A}, K_{A}^{\prime}, G_{A}^{\prime}$ converge to functions $F, K^{\prime}, G^{\prime}$ in the $\Lambda \rightarrow \infty$ limit is proved as in Sect. 4.2. Finally, $G \circ F$ is well defined in view of the exponential fall-off properties of $G, F$ and of the functions $S$ involved in $\circ$ and of the fact that singularities at short distances are at most logarithmic (apart from $\delta$ functions that can be integrated explicitly). The fact that $G_{\Lambda}{ }^{\circ}(\Lambda) F_{A}$ converges to $G \circ F$ can be obtained e.g. by decomposing $G \circ F-G_{A^{\circ} \circ{ }_{A} F_{A}}$ as $\left(G-G_{A}\right) \circ F$ $+G_{\Lambda}\left(\circ-{ }^{\circ}(\Lambda) F+G_{A_{A}}{ }_{\Lambda}\left(F-F_{\Lambda}\right)\right.$ : uniform bounds that include exponential fall-off factors in the distance between $u_{1}, \ldots, u_{4}$ and the boundary $\partial \Lambda$ of $\Lambda$ are again obtained.

We conclude with the following result:

Proposition 4 (Euclidean invariance). All functions $F, K, G$ obtained in the $A \rightarrow \infty$ limit are Euclidean invariant. Let us indicate the argument e.g. for $K$.

Let $\tau(x, y)$ be obtained from $(x, y)$ by a given Euclidean transformation $\tau$. Then $K_{\Lambda}(\tau(x, y))=K_{\tau^{-1} \Lambda}(x, y)$. On the other hand, the limits of $K_{\Lambda}$ and $K_{\tau^{-1} \Lambda}$ coincide in the $\Lambda \rightarrow \infty$ limit: in fact, the differences $K_{\Lambda}-K_{\tau^{-1} \Lambda}$ can be again bounded by uniform factors times exponential fall-off factors in the distance between $(x, y)$ and the union $\partial \Lambda \cup \partial\left(\tau^{-1} \Lambda\right)$.

\section{Momentum-Space Analyticity}

Proposition 5. $\forall \varepsilon>0, \exists \lambda_{\varepsilon}>0$ such that, for $|\lambda|<\lambda_{\varepsilon}$ :

1) The 2-point function $S$ is analytic in momentum-space in the region $s<3 m-\varepsilon$ apart from a pole at $s=\left(m_{\mathrm{ph}}(\lambda)\right)^{2} ; m_{\mathrm{ph}}$ is arbitrarily close to $m$ if $\lambda$ is sufficiently small.

2) $G\left(k ; z, z^{\prime}\right)$, where $k=p_{1}+p_{2}=p_{3}+p_{4}, z=\frac{p_{1}-p_{2}}{2}, z^{\prime}=\frac{p_{3}-p_{4}}{2}$, is analytic (and bounded) in a region of the form $k \in \Delta, z \in D, z^{\prime} \in D$, where $\Delta$ is a complex neighborhood of the set $k=\left(k_{0}, 0\right), k_{0} \leqq 4(m-\varepsilon)$ and $D=\left\{\left(z_{0}, z_{1}\right) ;\left|\operatorname{Re} z_{0}\right|<2(m-\varepsilon)\right.$, $\left.\left|\operatorname{Im} z_{1}\right|<\varepsilon\right\}$. 
Proof. By projection on the time axis, the exponential fall-off factors of the bounds (55), (56) yield fall-off factors $e^{-3(m-\varepsilon)\left|\left(u_{1}-u_{2}\right) 0\right|}$, respectively $e^{-m(1-\varepsilon) d\left(u_{1}, \ldots, u_{4}\right)}$, where $d$ is defined in Eq. (2). [A small part of the fall-off factors of (55), (56) can also be used to get fall-off factors in space variables.]

Property 2) of Proposition 5 follows directly from the Laplace transform theorem as already done in $[1,2]$.

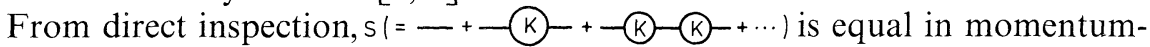
space to $\left[p^{2}+m^{2}-K(p)\right]^{-1}$, where by the Laplace transform theorem $K$ is analytic in the region $\operatorname{Re} p_{0}<3 m-\varepsilon^{\prime}$, and is moreover uniformly bounded there by $\operatorname{cste}\left(\varepsilon^{\prime}\right)|\lambda|$. Property 1) follows from invariance properties. Finally $m_{\mathrm{ph}}^{2}$, the zero of $p^{2}+m^{2}-K$, is arbitrarily close to $m^{2}$, e.g. via the implicit function theorem.

Acknowledgements. This work has benefitted from a partial collaboration of J. Bros and R. Sénéor. We also thank J. Feldman and V. Rivasseau for useful discussions.

\section{References}

0. Glimm, J., Jaffe, A., Spencer, T.: The Wightman axioms and particle structure in the $P(\varphi)_{2}$ quantum field model. Ann. Math. 100, 585-632 (1974)

1. a) Spencer, T.: The decay of the Bethe-Salpeter kernel in $P(\varphi)_{2}$ quantum field. Commun. Math. Phys. 44, 153-164 (1975)

b) Spencer, T.: Zirilli, F.: Scattering states and bound states in $\lambda P(\varphi)_{2}$. Commun. Math. Phys. 49, 1-16 (1976)

2. a) Glimm, J., Jaffe, A.: In the International Symposium on Mathematical Problem in Theoretical Physics. Lecture Notes in Physics, Vol. 39. Araki, H. (ed.). Berlin, Heidelberg, New York: Springer 1975

b) Glimm, J., Jaffe, A.: The resummation of one-particle lines. Commun. Math. Phys. 67, 267-293 (1979)

3. a) Combescure, M., Dunlop, F.: $N$-particle irreducible functions in Euclidean quantum field theory. Ann. Phys. 122, 102 (1979)

b) Combescure, M., Dunlop, F.: Three-body asymptotic completeness for $P(\varphi)_{2}$ models. Commun. Math. Phys. 85, 381 (1982)

c) Cooper, A., Feldman, J., Rosen, L.: Legendre transforms and $r$-particle irreducibility in quantum field theory; the formalism for $r=1$,2. Ann. Phys. 137, 146 (1981)

4. Dimock, J., Eckmann, J.-P.: Ann. Phys. 103, 289 (1977)

5. Bros, J.: In: Analytic methods in mathematical physics, p. 85. New York: Gordon and Breach 1970

6. Bros, J., Lassalle, M.: Analytic properties and many body structure in general quantum field theory, III. Commun. Math. Phys. 54, 33-62 (1977)

7. Bros, J., Iagolnitzer, D., Magnen, J., Sénéor, R.: In preparation

8. Iagolnitzer, D.: Irreducibility, analyticity, and unitarity of asymptotic completeness relations in massive quantum field theory. Fizika 17, 361 (1985)

9. Bros, J.: Physica 124 A, 145 (1984). Amsterdam: North-Holland 1978

10. Feldman, J., Magnen, J., Rivasseau, V., Sénéor, R.: The massive Gross-Neveu model in two dimensions. Commun. Math. Phys. 103, 67-103 (1986)

11. Duneau, M., Iagolnitzer, D., Souillard, B.: Decrease properties for classical lattices and continuous systems. Commun. Math. Phys. 31, 191 (1973); 35, 307 (1974)

12. Eckmann, J.-P., Magnen, J., Sénéor, R.: Decay properties and Borel summability for the Schwinger functions in $P(\varphi)_{2}$ theories. Commun. Math. Phys. 39, 259 (1974)

13. Gawedzki, K., Kupiainen, A.: Gross-Neveu model through convergent perturbation expansions. Commun. Math. Phys. 102, 1 (1986) 
14. Iagolnitzer, D.: Regularized and renormalized Bethe-Salpeter equations: some aspects of irreducibility and asymptotic completeness in renormalizable theories. Commun. Math. Phys. 99, 451 (1985)

Irreducibility and asymptotic completeness in renormalizable theories: Some remarks. Fizika 17, 3 (1985)

15. Bros, J., Iagolnitzer, D., Magnen, J.: In preparation

16. Symanzik, K.: Infrared simgularities and small distance behaviour analysis. Commun. Math. Phys. 34, 7 (1973)

17. Bros, J., Ducomet, B.: Two particle structure and renormalization. Ann. Inst. Henri Poincaré 45, 173 (1986)

18. Brydges, D.: A rigorous approach to Debye screening in dilute classical Coulomb systems. Commun. Math. Phys. 58, 313 (1978)

19. Battle, G., Federbush, P.: A phase cell cluster expansion for Euclidean field theories. Ann. Phys. 142, 95 (1982)

20. Glimm, J., Jaffe, A., Spencer, T.: In: Constructive quantum field theory. Velo, G., Wightman, A. (eds.). Berlin, Heidelberg, New York: Springer 1973

21. Nelson, E.: A quartic interaction in two dimensions. In: Mathematics theory of elementary particles. Goodman, R., Segal, I. (eds.). Cambridge: MIT Press 1966

22. Glimm, J., Jaffe, A.: A $\lambda\left(\varphi^{4}\right)_{2}$ quantum field theory without cut-off, I. Phys. Rev. 176, 1945 (1968)

23. Renouard, P.: Analyticité et sommabilité «de Borel» des fonctions de Schwinger du modèle de Yukawa en dimension $d=2$, I. Approximation «a volume fini.» Ann. Inst. Henri Poincaré 27, 237 (1977)

24. Feldman, J., Magnen, J., Rivasseau, V., Sénéor, R.: Construction and Borel summability of infrared $\varphi_{4}^{4}$ by a phase space expansion. Commun. Math. Phys. (in press) (1987)

Communicated by K. Osterwalder

Received June 13, 1986 